\title{
Mechanoreceptor synapses in the brainstem shape the central representation of touch
}

\author{
Brendan P. Lehnert ${ }^{1,2, \#}$, Celine Santiago ${ }^{1,2, \#}$, Erica L. Huey ${ }^{1,2}$, Alan J. Emanuel ${ }^{1,2}$, Sophia \\ Renauld $^{1,2}$, Nusrat Africawala ${ }^{1,2}$, llayda Alkislar ${ }^{1,2}$, Yang Zheng ${ }^{1,2}$, Ling Bai ${ }^{1,2}$, Charalampia \\ Koutsioumpa ${ }^{1,2}$, Jennifer T. Hong ${ }^{1,2}$, Alexandra R. Magee ${ }^{1,2}$, Christopher D. Harvey ${ }^{1}$, David \\ D. Ginty ${ }^{1,2, *}$ \\ ${ }^{1}$ Department of Neurobiology, Harvard Medical School, 220 Longwood Avenue, Boston, MA \\ 02115 \\ ${ }^{2}$ Howard Hughes Medical Institute, Harvard Medical School, 220 Longwood Avenue, Boston, MA \\ 02115
}

\section{Summary}

Mammals use glabrous (hairless) skin of their hands and feet to navigate and manipulate their environment. Cortical maps of the body surface across species contain disproportionately large numbers of neurons dedicated to glabrous skin sensation, in part reflecting a higher density of mechanoreceptors that innervate these skin regions. Here, we find that disproportionate representation of glabrous skin emerges over postnatal development at the first synapse between peripheral mechanoreceptors and their central targets in the brainstem. Mechanoreceptor synapses undergo developmental refinement that depends on proximity of their terminals to glabrous skin, such that those innervating glabrous skin make synaptic connections that expand their central representation. In mice incapable of sensing gentle touch, mechanoreceptors innervating glabrous skin still make more powerful synapses in the brainstem. We propose that the skin region a mechanoreceptor innervates controls refinement of its central synapses over development to shape the representation of touch in the brain.

\section{In Brief:}

Mechanoreceptors that innervate glabrous skin form more numerous and more powerful synaptic connections onto brainstem ascending projection neurons as compared to mechanoreceptors that

\footnotetext{
*Lead contact: david_ginty@hms.harvard.edu.

\#These authors contributed equally

Author Contributions

B.P.L., C.S., and D.D.G. conceived the study. B.P.L. performed functional imaging and electrophysiological experiments with assistance from E.H., A.J.E, S.R., and C.D.H.; C.S. performed anatomical experiments with the assistance of N.A., S.R., B.P.L., I.A., Y.Z., L.B., C.K., J.T.H. and A.R.M.; B.P.L., C.S., and D.D.G. wrote the paper with input from all authors.

Declaration of Interests

The authors declare no competing interests.

Publisher's Disclaimer: This is a PDF file of an unedited manuscript that has been accepted for publication. As a service to our customers we are providing this early version of the manuscript. The manuscript will undergo copyediting, typesetting, and review of the resulting proof before it is published in its final form. Please note that during the production process errors may be discovered which could affect the content, and all legal disclaimers that apply to the journal pertain.
} 
innervate hairy skin. This suggests that skin type affects how neurons in the brain represent neurons in the body.

\section{Graphical Abstract}

The proportion of central neurons representing glabrous

skin expands over postnatal development...

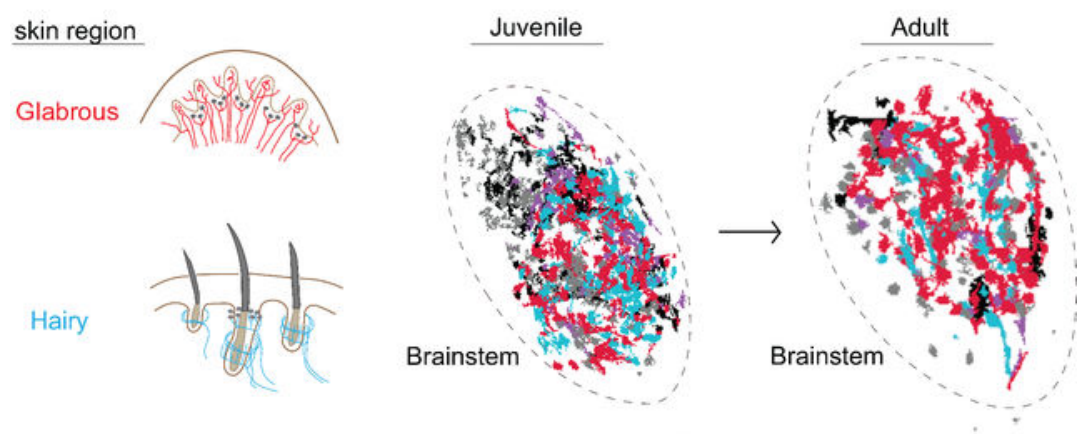

...reflecting differences in developmental synaptic refinement that are skin region dependent.

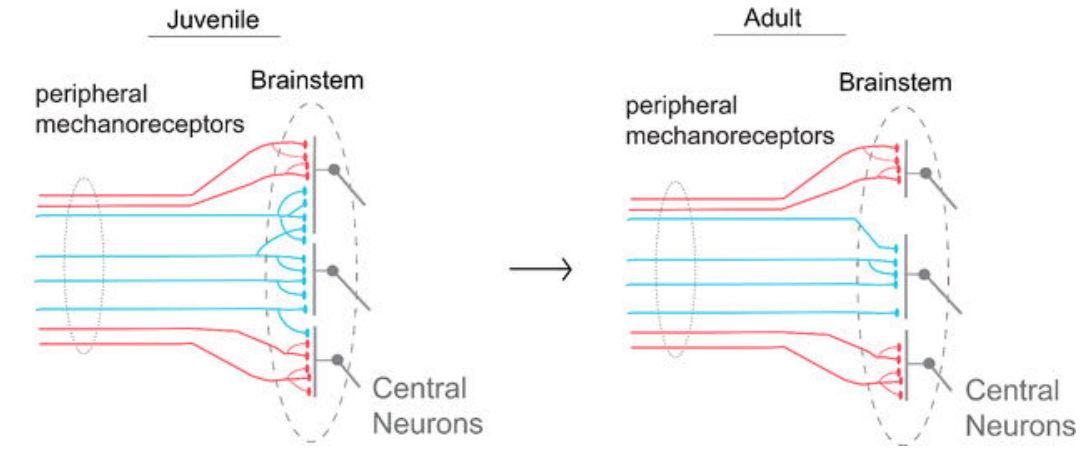

\section{Introduction}

Perception relies on populations of sensory neurons that represent salient features of the physical world. Neurons are not uniformly allocated across a sensory space, but rather, particular regions are often represented by disproportionate numbers of neurons. A striking example is the primate visual system, where neurons across the visual hierarchy disproportionately represent central regions of the visual field, in part reflecting the high density of photoreceptors in the center of the primate retina (Cowey and Rolls, 1974). Disproportionate central representation of foveal visual space has far-reaching consequences, as it shapes the ocular motor strategies used to survey the world as well as cognitive strategies used to comprehend visual scenes (Yarbus, 1967). Similarly, the human somatosensory cortex contains an iconic map of the body, the homunculus, that does not faithfully reflect our physical form, but rather disproportionately allocates neurons to the hands and face relative to the torso and proximal limbs (Penfield and Boldrey, 1937). Disproportionate central representation may reflect the tension between completely tiling a sensory space and allocating neural resources to areas where acuity or representational capacity is critical. 
A common feature of mammalian cortical body maps is that they favor glabrous (nonhairy) skin surfaces (Penfield and Boldrey, 1937; Sur et al., 1978; Sur et al., 1980; Xu and Wall, 1999). For example, glabrous skin representations occupy $30 \%$ of primate somatosensory cortex but only $5 \%$ of skin surface area (Sur et al., 1982). The distortions to figure of the cortical somatosensory homunculus may reflect differences in the density of mechanoreceptors in skin across the body (Catani, 2017). This view is supported by observations in rodents, where neurons in layer IV of somatosensory cortex that respond to whisker stimulation are organized into barrels, and anatomical measurements revealed that the number of neurons in each barrel is proportionate to the number of myelinated sensory neurons innervating the corresponding whisker (Lee and Woolsey, 1975).

However, multiple lines of evidence challenge the conventional view that disproportionate central representation of the body is simply a reflection of skin innervation density. For example, the primary somatosensory cortex and the superior colliculus contain body maps with differing proportions (Drager and Hubel, 1976), suggesting that the same peripheral inputs can support different central representations. Moreover, a compilation of human anatomical and physiological measurements argues that differences in peripheral sensory neuron numbers may not account for the over-representation of the hands and the digits in somatosensory cortex (Corniani and Saal, 2020). This parallels work in the visual system demonstrating that photoreceptor density at the primate retinal fovea is insufficient to explain the enlarged representation of central fields of view in the visual cortex (Azzopardi and Cowey, 1993). Thus, despite the prevalence of disproportionate body maps across mammals with diverse body forms, the mechanisms that shape the representation of the body in the brain have remained incompletely understood since the discovery of these maps nearly a century ago.

Here, we examine body representation in mice and find that disproportionate emphasis of glabrous skin emerges during postnatal development in multiple brain areas, while innervation density remains stable. This shift reflects developmental refinement of synaptic connections in the brainstem and the skin region a mechanoreceptor innervates. Functionally, optical stimulation of glabrous skin innervating mechanoreceptors evokes larger synaptic currents and is more effective at driving spiking in brainstem neurons than stimulation of hairy skin innervating sensory neurons. Interestingly, this difference is preserved in mice that do not sense gentle touch on their body surface. Together, our findings support a model in which peripheral innervation density and skin region dependent synaptic expansion in the brainstem act multiplicatively to shape the central representation of touch.

\section{Results}

\section{Disproportionate expansion of glabrous skin representation emerges over postnatal development while receptor density in the skin remains stable}

We investigated the development of glabrous skin representation in mouse primary somatosensory cortex (S1). Hindlimb and forelimb S1 were identified through retrograde labeling of corticospinal projections to the lumbar and cervical enlargements of the spinal cord (Figure 1A,B, Supplemental Figure 1). Stereotactic recordings spanning hindlimb and 
forelimb S1 were performed with a grid of multielectrode array (MEA) penetrations of S1 in lightly anesthetized mice. Recordings from hindpaw regions of P14 cortex showed an equal number of S1 neurons responding to paw glabrous and paw hairy skin stroke, while forepaw cortex emphasized paw glabrous skin surfaces (Figure 1B, C). Similar recordings in adult mice uncovered a more pronounced glabrous representation bias, an approximately 3 -fold increase in the number of neurons that exhibit paw glabrous-to-hairy skin preference across both hindpaw and forepaw S1, compared to P14 animals (Figure 1B, C). As functional responses in primate $\mathrm{S} 1$ depend on $\mathrm{A} \beta$-LTMRs that ascend the dorsal column of the spinal cord and project directly to the brainstem (Jain et al., 1997), we recorded from glabrous and hairy skin innervating A $\beta$-LTMRs and confirmed that skin stroke similarly excites both populations (Supplemental Figure 1). Thus, over-representation of paw glabrous skin relative to paw hairy skin dramatically increases from P14 to adulthood.

In the visual system, the heightened photoreceptor density characteristic of the primate fovea emerges over weeks of postnatal development (Packer et al., 1990), and so we next asked whether a similar developmental rearrangement of peripheral touch neurons accompanies the dramatic developmental shift in paw glabrous skin and hairy skin functional representation observed in S1. We measured the distribution of the terminal endings of A $\beta$-LTMRs in the skin through retrograde viral transduction of sensory neurons that ascend the dorsal column, using the Cre-dependent alkaline phosphatase (AP) reporter mouse Brn3a ${ }^{c K O A P}$ (Figure 1D; Badea et al., 2009b) in P14 and adult (P50) mice. Viral transduction was titrated such that dorsal column-projecting A $\beta$-LTMRs were sparsely labeled and the endings of individual neurons in the skin were distinguishable (see Methods, Supplemental Figure 1); all known A $\beta$-LTMR subtypes were detected in these experiments (Wu et al., 2012; Bai et al., 2015). There are stark differences in the number of A $\beta$-LTMRs/ skin area along the proximal-distal axis of the limbs of adult mice, consistent with previous electrophysiological and anatomical measurements in other species (Wang et al., 1997) (Supplemental Figure 1). Additionally, at both P14 and adulthood, forepaw glabrous skin is approximately three times more densely innervated by A $\beta$-LTMRs than forepaw hairy skin, consistent with prior functional measurements in mice (Walcher et al., 2018) (Figure 1D, E). In contrast, hindpaw glabrous and hindpaw hairy skin have close to equal innervation densities at these two ages (Figure 1D, E). For each paw, the ratio of innervation densities is expressed as a glabrous: hairy ratio (Figure 1E, F). Between P14 and adulthood, we detect no significant changes in glabrous: hairy skin A $\beta$-LTMR innervation ratios in either the forepaws or the hindpaws (Figure 1E). We measured the peripheral arbor morphologies of sparsely labeled neurons in glabrous and hairy skin and found no changes in terminal area or number between P14 and adulthood (Supplemental Figure 1).

These functional and anatomical findings define the relationship between paw glabrous and paw hairy skin innervation density and central (S1) representation over development. At $\mathrm{P} 14$, paw glabrous skin and hairy skin representation in $\mathrm{S} 1$ is proportionate to A $\beta$-LTMR skin innervation density (Figure $1 \mathrm{~F}$ ). In contrast, in adults, there is an approximately 3-fold expansion of glabrous skin preferring $\mathrm{S} 1$ neurons beyond that predicted by the distribution of A $\beta$-LTMRs. We also recorded from the external nucleus of the inferior colliculus, another brain region that exhibits a prominent representation of the body surface (Figure 1F). Inferior collicular representations of glabrous skin in adult mice exceed that predicted by 
the relative numbers of LTMR endings across hindpaw skin, similar to S1. Taken together, these findings demonstrate that the degree of central representation of paw glabrous skin and paw hairy skin surfaces is consistent with receptor density early in postnatal development, but that there is a dramatic and unexplained expansion of cortical and collicular neurons responding to touch of glabrous skin in adulthood.

\section{In vivo multiphoton imaging of neurons in the gracile nucleus of the brainstem reveals a locus of expansion}

Developmental increases in the number of glabrous skin preferring neurons in cortex (S1) and inferior colliculus could reflect changes at a common anatomical locus earlier in the somatosensory hierarchy. Therefore, we performed dual retrograde labeling experiments targeting the ventroposteriolateral (VPL) thalamus (the main input to somatosensory cortex) and the inferior colliculus to visualize cell bodies of ascending projection neurons of both the gracile nucleus $(\mathrm{GN})$ and cuneate nucleus $(\mathrm{CN})$ of the brainstem dorsal column nuclei, a region that receives direct synaptic input from A $\beta$-LTMRs. Dual-color retrograde labeling in $\mathrm{Gad} 2^{n l s-m c h e r r y}$ mice demonstrated the presence of substantial, largely nonoverlapping and intermingled populations of thalamic projection neurons, inferior collicular projection neurons, and presumptive local inhibitory neurons within both the GN and $\mathrm{CN}$ (Supplemental Figure 2). We developed a preparation for imaging the GN using multiphoton microscopy in mice where GN thalamic projection neurons were retrogradely transduced with the calcium indicator jGCaMP7s (Figure 2A). The GN, which encodes mechanosensory inputs from the caudal half of the animal, including the hindlimbs, is optically accessible in acute preparations that expose the brainstem, while the $\mathrm{CN}$ is not because it lies beneath ascending fiber tracts. Tactile responses of hundreds of GN neurons at depths up to $\sim 250 \mu \mathrm{m}$ and throughout the entirety of the GN were routinely observed for up to six hours (Figure 2A, B).

We next asked whether disproportionate representation of glabrous skin is present within the GN and if so, whether it emerges over postnatal development. Stroke to the ipsilateral hindpaw glabrous skin, hindpaw hairy skin, thigh skin, or back skin of P14 mice (equivalent, approximately $10 \mathrm{~mm}^{2}$ areas) evoked robust responses in GN neurons (Figure 2B). Spatial maps of the body revealed a core region of intermingled hindpaw glabrous skin and hairy skin preferring neurons and less numerous populations of thigh and back hairy skin preferring neurons (Figure 2D). At P14, GN thalamic projection neurons showed no preference at the population level for touch to glabrous skin compared to hairy skin of the hindpaw (Figure 2B, D). The same was true for GN inferior collicular projecting neurons, which exhibited no population bias towards glabrous skin at P14 (Figure 2E).

In contrast, in adult mice approximately three times as many GN thalamic projection neurons preferentially responded to glabrous skin stroke as those that preferentially responded to stroke on a comparable surface area on the hairy skin side of the same paw (Figure 2F, H). GN inferior collicular projecting neurons also showed a marked preference for glabrous skin stimulation (Figure $2 \mathrm{G}, \mathrm{H}$ ), while glabrous skin representation exceeding skin innervation densities were not observed for locally projecting inhibitory neurons (Figure $2 \mathrm{H}$ ). GN thalamic projection neuron populations had glabrous skin to hairy skin 
preference ratios that were equivalent to cortical (S1) neuron preference ratios measured at both P14 and adult (Figure 2I). Taken together, these findings indicate that disproportionate representation of glabrous skin in both $\mathrm{S} 1$ and the inferior colliculus is partially or fully driven by changes in the GN over development.

\section{The axonal and synaptic structure of LTMR projections to brainstem reflects the skin region innervated and developmental stage}

Disproportionate central representation of glabrous skin might reflect developmental changes in A $\beta$-LTMR central projections and their synapses onto brainstem neurons. Therefore, we next sparsely labeled A $\beta$-LTMRs to explore the relationship between the skin type innervated and the branching patterns of their axonal arbors in the GN and $\mathrm{CN}$. The three main classes of cutaneous A $\beta$-LTMRs were stochastically labeled using the Cre-dependent AP reporter mouse $B r n 3 a^{c K O A P}$ in conjunction with LTMR-selective CreER lines and low-dose tamoxifen treatments (see Methods). Samples had 1-4 labeled LTMRs per animal with central projections terminating in the GN or $\mathrm{CN}$, such that the peripheral arbors of individual LTMRs could be identified in the skin based on established somatotopy. This approach was effective in generating sparsely labeled trunk-level A $\beta$-LTMRs, but more rarely resulted in the labeling of paw-innervating LTMRs, likely because they are fewer in number, as the majority of the mouse's skin consists of trunk hairy skin. Thus, we generated a dual Cre and FlpO recombinase-dependent Tau ${ }^{F S-i A P}$ reporter mouse to intersectionally label neurons through injection of an AAV2/1-FlpO virus to the skin of animals containing CreER alleles (Figure 3A and Supplemental Figure 3).

We reconstructed the entire axonal arbors of each A $\beta$-LTMR type that innervates paw glabrous skin, paw hairy skin, or trunk hairy skin (Figure 3 and Supplemental Figure 3). Paw glabrous skin-innervating A $\beta$-LTMRs form elaborate central arbors in the GN or $\mathrm{CN}$ that dwarf those formed by trunk level A $\beta$-LTMRs and by the majority of paw hairy skin-innervating A $\beta$-LTMRs (Figure 3B-C, Supplemental Figure 3). Intriguingly, the central arbors of a small number of paw hairy skin-innervating LTMRs were equally as complex as those of glabrous skin-innervating LTMRs (Figure 3B-C). To determine if this variability in hairy LTMR central arbor structure is explained by LTMR subtype or location, we identified the exact position and morphology of each neuron's peripheral arbor within the paw skin. We detected no relationship between the genetic class of neurons and the properties of their central arbors (Supplemental Figure 3). Strikingly, hairy skin-innervating neurons with peripheral arbors located at the border between paw glabrous skin and paw hairy skin $(<0.5$ $\mathrm{mm}$ from the edge of the paw after dissection) form arbors in the $\mathrm{GN}$ or $\mathrm{CN}$ that are significantly more elaborate than those formed by LTMRs innervating hairy skin $>0.5 \mathrm{~mm}$ from the edge of the paw (Figure 3B-C, Supplemental Figure 3). One such labeled neuron formed peripheral endings that span both hairy and glabrous skin, and this neuron formed a complex central arbor in the brainstem that resembled those of glabrous skin-innervating neurons (Figure 3C, arbor indicated by asterisks). Together, these findings demonstrate that the proximity of an A $\beta$-LTMR's peripheral arbor to glabrous skin predicts the complexity of its axonal arbors in the brainstem across multiple genetically and morphologically distinct LTMR subclasses. 
We also explored the relationship between A $\beta$-LTMR skin innervation sites and their histologically-defined synapses in the brainstem. Presynaptic terminals of A $\beta$-LTMRs were labeled with Synaptophysin-tdTomato or Synaptophysin-mScarlet fusion proteins, hereafter referred to as syn-fluorescent protein (syn-FP), by injecting AAVs into the glabrous or hairy skin of the paw or trunk (Figure 4A). We quantified the number of syn- $\mathrm{FP}^{+}$puncta in the GN or $\mathrm{CN}$ and the number of labeled LTMR axons entering the brainstem to calculate average synapse numbers per LTMR. In GN and CN sections co-stained with synaptic markers, the majority of syn- $\mathrm{FP}^{+}$puncta were found in apposition to Homer1, a post-synaptic marker for excitatory synapses, and co-localized with vGlut1, a presynaptic marker for A $\beta$-LTMRs (Figure 4A and Supplemental Figure 4). This analysis revealed that individual adult forepaw glabrous skin innervating neurons form more synapses in the $\mathrm{CN}$ than adult forepaw hairy skin innervating neurons (Figure 4B). Similarly, adult hindpaw glabrous skin innervating neurons form more synapses in the GN than adult neurons innervating back, thigh, and hindpaw hairy skin (Figure 4C).

To determine when these synaptic differences in A $\beta$-LTMR central arbors emerge over development, we injected AAV-syn-FP into hindpaw glabrous skin, hindpaw hairy skin, or thigh hairy skin to label A $\beta$-LTMRs in P14 animals. At P14, hindlimb hairy skinand glabrous skin-innervating A $\beta$-LTMRs form similar numbers of synapses per neuron, in contrast to adult timepoints (Figure 4C). Both hindlimb hairy skin- and glabrous skin-innervating A $\beta$-LTMRs form more synapses in the GN at P14 than observed in adulthood (Figure $4 \mathrm{C}$ ). However, this difference is more pronounced for hindpaw hairy skin-innervating A $\beta$-LTMRs, which exhibited a $~ 75 \%$ loss of synapses per neuron between P14 and adulthood. Similarly, we found that P5-P10 hairy skin-innervating and trunk-level A $\beta$ RA-LTMRs form significantly more complex axonal arbors in the GN or CN compared to their adult counterparts when quantifying the total number of end points (Supplemental Figure 3).

We next examined the receptive fields of GN thalamic projection neurons at postnatal and adult timepoints (Supplemental Figure 4). We found that these receptive fields decline in size over time, consistent with developmental refinement and possible pruning of synaptic connections. Fewer GN thalamic projection neurons with large, multiregional receptive fields were observed as development progressed (Supplemental Figure 4). Glabrous expansion could occur through an increase in the number of GN neurons responding to glabrous touch, or a depletion of those responding to hairy skin stimulation. We observed a developmental decline in the percentage of the GN thalamic projection neuron population that responded to hairy skin, but not glabrous skin (Supplemental Figure 4). Anatomically, paw glabrous skin-innervating A $\beta$-LTMRs make more synapses in the brainstem compared to paw or trunk hairy skin-innervating LTMRs, and these differences emerge over postnatal development (Figure 4D). Therefore, disproportionate central representation of glabrous skin could emerge developmentally through skin region dependent differences in A $\beta$-LTMR synaptic refinement (Figure 4D). 


\section{Single spikes from glabrous skin-innervating LTMRs more powerfully excite GN neurons}

The functional connectivity of neurons in the visual system has been defined at single photoreceptor resolution through approaches that relate the history of spiking to a white noise stimulus in a neuron's receptive field (Field et al., 2010). To apply this approach, we expressed the light-gated ion channel ReaChR (Hooks et al., 2015) in peripheral neurons and optically mapped the glabrous and hairy skin receptive fields of GN neurons. Whereas mechanical stimuli that reliably drive spiking inevitably excite many A $\beta$ LTMRs in the skin, optogenetic approaches have the potential to selectively and reliably excite single LTMRs. We constructed an optomechanical device capable of rapidly and precisely exciting the endings of neurons in the skin of Cdx2-Cre; AviFFlpO; Rosa26LsL-FsF-ReaChR mice, which express ReaChR in all sensory neurons innervating hindlimb skin. We inserted MEAs into the L4 DRG and optically stimulated ReaChR expressing sensory neuron terminals in the skin with focused pulses of laser light, moving the stimulus to a new random location on the paw every millisecond (Figure 5A). We then used reverse correlation analysis to recover a time-varying spatial receptive field for individual units through computation of the spike-triggered average (Figure 5B, bottom). The optical receptive fields generated in this manner resemble the mechanosensory terminal fields of individual LTMRs and overlapped with their mechanical receptive fields (Figure 5B, n=11 neurons with targeted mechanical stimulation, data not shown, see Methods). Importantly, optical stimulation of A $\beta$-LTMRs in both hairy and glabrous skin typically produced single spikes with equivalent probabilities and latencies (Figure 5C, data not shown).

Next, identical optical stimuli were used to excite neurons innervating hindpaw glabrous skin, hindpaw hairy skin, or thigh while recording from the GN of the brainstem using an MEA targeted to the GN (Figure 5D). In contrast to the LTMRs recorded in the DRG (Figure 5A-C), GN unit spike-triggered averages demonstrated complex spatiotemporal receptive fields with multiple, discontinuous receptive field "spots" (RF spots) that were reminiscent of individual LTMR spatial receptive fields and staggered with respect to one another in time (Figure 5E, Supplemental Figure 5C-D). RF spots had similar strength and sizes in control experiments where only a fraction of A $\beta$-LTMRs expressed ReaChR, though the number of spatiotemporally isolated RF spots identified were fewer, as expected if each RF spot reflects the contribution of a single LTMR input to a GN neuron's receptive field (Supplemental Figure 5E, F).

Examination of the time-projected spatial receptive fields of optically-evoked GN units demonstrated that stimulation of a single RF spot - which likely corresponds to a single A $\beta$-LTMR input - in glabrous skin is more effective at driving spiking in GN neurons than a similar stimulus applied to a single hairy skin RF spot (Figure 5E, F). This effect was consistent across 120 spatiotemporally well-isolated RF spots identified across eight animals (Figure 5F). Because the optical stimuli used for these GN maps are equally effective at exciting glabrous skin and hairy skin LTMRs, based on our DRG recordings (Figure 5C), this finding indicates that stimulation of individual glabrous skin-innervating A $\beta$-LTMRs more strongly evokes spiking in GN neurons compared to hairy skin-innervating A $\beta$-LTMRs. 


\section{Fiber fraction measurements of synaptic strength and convergence reveal skin region dependent differences in LTMR inputs to thalamic PNs in the GN}

Our histological synaptic analysis (Figure 4) and optogenetic receptive field measurements (Figure 5) suggest that glabrous skin innervating LTMRs make more powerful synaptic connections onto GN neurons. However, the in vivo optogenetic receptive field experiments provide only an indirect measure of synapse strength that lacks cellular specificity. To directly characterize synaptic connections between glabrous skin A $\beta$-LTMRs or hairy skin A $\beta$-LTMRs and GN thalamic projection neurons, we made whole-cell patch clamp recordings from GN thalamic projection neurons in acute brainstem slices. The relative strength of glabrous skin and hairy skin innervating A $\beta$-LTMR synapses was measured by optogenetically exciting single presynaptic axons in $T r k B^{C r e E R}$; AviFFlpO; $R 26^{L S L-F s F-R e a C h R:: m C i t r i n e}$ mice that expressed ReaChR in A $\beta$ RA-LTMRs that innervate glabrous skin, or Ret ${ }^{C r e E R}$; Pvalb ${ }^{F l p O}$; R26 $6^{L S L-F-C h R 2:: Y F P-F}$ mice that expressed ReaChR in A $\beta$ RA-LTMRs that innervate hairy skin (see Methods). To measure the total ascending synaptic input and produce corresponding fiber fraction estimates of the convergence of presynaptic inputs onto GN thalamic projection neurons, we also electrically stimulated the dorsal column with a bipolar electrode (Hooks and Chen, 2006; Litvina and Chen, 2017).

GN thalamic projection neurons that had been retrogradely labeled from the thalamus with CTB-647 were visually targeted for whole cell patch clamp recordings using a horizontal brainstem slice preparation. Minimal optical stimulation of A $\beta$ RA-LTMRs produced shortlatency unitary synaptic EPSCs interspersed with failures (Figure 6B, E, left panels). EPSC waveforms were stereotyped, and histograms of the evoked synaptic current amplitudes across multiple trials were bimodal, consistent with single fiber isolation (Figure 6B, E, right panels). Maximal EPSCs evoked through saturating electrical stimulation of dorsal column axons were of comparable amplitude across recordings from GN thalamic projection neurons postsynaptic to glabrous skin- and hairy skin-innervating LTMRs (Figure 6C, F, Mann Whitney $U$ test $p>0.05$ ). Immunohistochemical visualization of genetically labeled LTMRs and filled, recorded GN thalamic projection neurons revealed regions of presynaptic (fluorescent protein and VGlut1+) and postsynaptic, dendritic apposition, consistent with a monosynaptic connection (Figure 6D, G).

These GN whole-cell recordings revealed that glabrous skin-innervating A $\beta$ RA-LTMR single fiber strengths are two-fold larger than hairy A $\beta$ RA-LTMR fiber strengths (Figure $6 \mathrm{H})$. Moreover, our electrical stimulation findings show that "glabrous skin" and "hairy skin" GN thalamic projection neurons receive comparable total synaptic input (Figure $6 \mathrm{C}, \mathrm{F})$. Skin region specific estimates of the number of presynaptic ascending neurons that converge onto individual GN thalamic projection neurons were computed by dividing the maximal electrically-evoked EPSC by the minimal optogenetic single fiber EPSC (Figure 6I). Together, these findings support a model in which glabrous skin-innervating LTMRs make stronger and less convergent synaptic connections onto GN thalamic projection neurons compared to hairy skin-innervating LTMRs (Figure 6J). 


\section{Disproportionate glabrous skin representation is maintained in Piezo2 conditional knockout mice}

In the visual system, both spontaneous and visually evoked patterns of retinal activity contribute to pruning of retinal ganglion cell synapses in the thalamus during postnatal development (Hooks and Chen, 2006). To determine if touch evoked activity in LTMRs shapes the relative strength of A $\beta$-LTMR synapses in the brainstem, we analyzed conditional knock-out animals in which Piezo2, the principal mechano-sensitive ion channel for LTMRs (Ranade et al., 2014), is deleted using the $C d \times 2$-Cre line, which drives recombination broadly in neurons below cervical level $\mathrm{C} 2$, including DRG neurons. Cdx2-Cre; Piezo $2^{\text {flox/flox }}$ and Cdx2-Cre; Piezo $2^{\text {flox/Null }}$ animals display severe coordination deficits, similar to those reported for Hoxb8-Cre; Piezo2 cKO animals (Woo et al., 2015; Murthy et al., 2018). In situ hybridization experiments in Cdx2-Cre; Piezo2flox/flox animals demonstrate efficient deletion of the floxed Piezo2 coding sequence in lumbar-level DRG neurons (Supplemental Figure 7). We assessed touch sensitivity and optical excitability of A $\beta$-LTMRs that lack Piezo2, with the goal of comparing optical receptive fields from different skin regions in GN neurons in brainstem.

In wild type animals that express ReaChR in all sensory neurons, many of the DRG units that spike in response to optical stimulation of skin also respond to innocuous mechanical brushing, indentation, or pulling of the glabrous skin that mimics gripping forces. This is an underestimate of the extent of mechanosensitivity within the population, as individual receptive fields were not explicitly targeted (Figure 7A). In contrast, light touch evoked responses are nearly absent in Cdx2-Cre; Piezo2 $2^{\text {flox/flox; }}$ AviFFlpO; R26-FsF-ReaChR animals, while responses to optical stimulation and strong pinch persisted (Figure 7B). GN thalamic PNs in Piezo2 conditional mutants showed a similar, nearly complete loss of innocuous touch responses, with no change in the number of neurons responsive to a strong pinch stimulus (Supplemental Figure 7), excluding the possibility of latent, indirect mechanosensory input from small caliber LTMRs that might not be easily detected in MEA recordings of DRG neurons. Importantly, the number of DRG sensory neurons responding to skin optical stimulation was equivalent between Piezo2 conditional mutants and control animals (Figure 7C).

GN neurons recorded in $C d x 2-C r e ; P i e z o 2^{\text {flox/flox }} ;$ Avil FlpO; R26 FsF-ReaChR Piezo2 conditional mutant mice had spatiotemporally well-isolated single RF spots with dimensions reminiscent of individual LTMRs (similar to the experiments presented in Figure 5). These isolated RF spots most likely represent single LTMR inputs to GN neurons (Figure 7E). Similar to control animals, we found that stimulation of individual RF spots on glabrous skin more strongly excited GN neurons than comparable stimuli applied to hairy skin in the Piezo2 mutant mice that lack mechanically-evoked LTMR activity (Figure 7E, F). Together, these findings indicate that the disproportionate strength of glabrous skin-innervating LTMR synapses in the brainstem can be established independent of mechanically-evoked activity in LTMRs. 


\section{Discussion}

Mammalian nervous systems are embedded in a fascinating diversity of body forms that in turn shape their structure, and nearly half of the mouse brain contains neurons that are organized with respect to the body (Wang et al., 2020). Glabrous skin responsive neurons occupy disproportionately large cortical regions in primates, cats, squirrels, and bats, suggesting a common mechanism might unite these observations (Sur et al., 1978; Sur et al., 1980). Here, we discover a stereotyped transformation during postnatal development that expands the proportion of central neurons dedicated to glabrous skin and demonstrate that remapping occurs at the first synapse between peripheral mechanoreceptors and their central targets in the brainstem.

\section{Determinants of body representation in the central nervous system}

Synaptic expansion, the ratio of presynaptic neurons to postsynaptic neurons in a neural circuit, will increase as convergence declines if other properties of the circuit remain stable (Litwin-Kumar et al., 2017). We show that convergence at LTMR-PN brainstem synapses depends on the skin region innervated, and propose that differential synaptic expansion enhances the central representation of glabrous skin. Within a skin region, representation may be proportionate to innervation density (Lee and Woolsey, 1975), but each skin region uniquely instructs the extent of expansion of mechanoreceptor synapses in the brainstem to disproportionately shape a collective central representation. This model reconciles previously observed discrepancies between innervation density and central representation across the body in multiple species (Catania and Kaas, 1997; Corniani and Saal, 2020; Lee and Woolsey, 1975).

Skin region dependent patterns and strengths of LTMR synaptic connections within the brainstem may reduce evolutionary constraints by flexibly tailoring body maps to disparate body forms. In the visual system, allometric scaling laws that relate the size of visual thalamus and cortex across primate species suggest that synaptic expansion enables higher order sensory areas to explicitly represent new features of a stimulus (i.e. direction of stimulus movement) while maintaining topography (Stevens, 2002). It is possible that expanded glabrous skin representation in the brainstem may support the subcortical emergence of neural feature tuning. Alternatively, parallel feature computations in early somatosensory circuits are proposed to increase the speed at which the nervous system can act on mechanical feedback (Tuthill and Wilson, 2016), such that disproportionate representation of glabrous skin might support parallel streams of rapid feedback underlying dexterous object manipulation.

\section{The development of body representation}

In star-nosed moles, a star-shaped pattern of cytochrome-oxidase densities in the brainstem forms over development, with the behaviorally most-relied upon regions of the star appendage forming first and later occupying a disproportionate area (Catania and Kaas, 1997b; Catania, 2001). In this system, disproportionate body representation may reflect an advantage that the earliest axonal arbors to arrive to the brainstem have over later arrivals, with corresponding potential roles for neural activity differences (Catania, 2001). 
These compelling models do not account for the disproportionate representation of glabrous skin relative to hairy skin reported here, as 1) glabrous skin and hairy skin innervating peripheral neuron axonal brainstem projections have similar numbers of synapses at P14 and 2) Piezo 2 mutant mice retain the relationship between skin target identity and the strength of LTMR synaptic outputs in the brainstem. Peripheral targets play key roles in controlling somatosensory neuron maturation, and skin type can instruct basic properties of peripheral neuron identity, such as the neurotransmitter they produce (Schotzinger and Landis, 1988; Luo et al., 2007). We propose that the skin region a mechanoreceptor innervates controls refinement of its central synapses over development to shape the representation of touch in the brain.

The proximity of a LTMRs mechanosensitive endings to glabrous skin is tightly associated with the expansiveness of its central axonal arbor in the brainstem, suggesting the possibility of a diffusible factor or cue intrinsic to glabrous skin that influences refinement of synaptic connections during postnatal developmental. This mechanism may extend beyond the central representations of gentle touch to the skin, as a recent study reported that the central arbor morphologies of a subset of nociceptors also vary by body location (Olson et al., 2017). Somatotopy develops in an outward-to-inward fashion, such that body maps established at lower levels of the somatosensory hierarchy drive the organization of higher regions (Iwasato and Erzurumlu, 2018). Extending this idea, we propose that, in addition to skin innervation density, skin target regions themselves are key determinants of disproportionate body representation in the developing brain.

\section{Limitations of the Study}

This study raises several questions, some of which we highlight here. First, while we focus on skin region-dependent developmental shifts at A $\beta$-LTMR-PN synapses in the brainstem, postsynaptic dorsal column (PSDC) neurons in the spinal cord also project to the brainstem. PSDCs may shape body representation and contribute to the heterotypic convergence estimates (Figure 2, Figure 5). Second, we show that skin region-dependent differences are preserved in Piezo2 mutant animals that do not sense gentle touch, but cannot rule out a potential role for noxious tactile stimuli or other modalities (i.e. thermal) in the establishment of skin region dependent A $\beta$-LTMR synaptic strength (Figure 7). Finally, we speculate that the ventral paw/glabrous skin either i) instructs developing LTMRs to form more synaptic connections in the brainstem or ii) generates cues that attract LTMRs that are fated to make more synaptic connections in the brainstem, but we do not identify any such factors here. The mechanisms instructing differences in synaptic properties of mechanosensory neurons whose endings are embedded in mammalian glabrous and hairy skin are not understood and will require further experimental insight.

\section{STAR $\star$ Method \\ RESOURCE AVAILABILITY}

Lead Contact-Further information and requests for resources should be directed to and will be fulfilled by David Ginty (david_ginty@hms.harvard.edu). 
Materials Availability-Requests for mouse lines generated in this study should be directed to and will be fulfilled by David Ginty (david_ginty@hms.harvard.edu).

\section{Data and Code Availability}

- $\quad$ All data reported in this paper will be shared by the lead contact upon request.

- All original code is available in this paper's supplemental information.

Quantification and Statistical Analysis-Statistical tests were conducted in Python 3.7.9 using the SciPy stats module or in GraphPad Prism. Non-parametric tests and parametric tests were used for comparing two independent groups (Mann-WhitneyWilcoxon test or Welch's t-test), two related groups (Wilcoxon signed-rank test), and multiple groups (Kruskal-Wallis test with Bonferroni correction). $\mathrm{p}<0.05$ was considered significant. Additional details on sample sizes and statistical tests for each experiment can be found in figure legends and the main text.

\section{EXPERIMENTAL MODEL AND SUBJECT DETAILS}

All mice used in the study are of mixed background. The dual recombinase-dependent Tau $^{\text {FSFiAP }}$ knock-in allele was generated at the Janelia Campus Research Gene Targeting and Transgenic Facility using standard ES cell targeting. Briefly, a FRT-2XSTOPFRT-5'PLAP-loxP-3'PLAP-loxP-attB-Neo-attP cassette was introduced by homologous recombination into the Tau (Mapt) gene, replacing the first ATG of the Tau coding sequence. The PLAP coding sequence encodes human placental alkaline phosphatase and its 3 ' half is inverted and flanked by head-to-head loxP sites, preventing expression in the absence of Cre recombination. TauFSFAP heterozygous mice were generated by mating chimeric males to females constitutively expressing PhiC31(R26-PhiC31o, JAX $\# 007670)$ to remove the neomycin selection cassette. Successful excision of the Neo cassette was confirmed in the next generation by PCR genotyping. The following primers were used to genotype TauFSFAP animals for the presence of the reporter allele: forward 5'-GGATGGGAAACTGAGGCTCT-3'; reverse 5'-ATGGTGGCGAATTCCAAATCA- 3'. $R 26^{F S F-R e a C h R}$ mice were generated from $R 26^{F S F-L S L-R e a C h R}$ mice (JAX \# 024846)(Hooks et al., 2015) through germline excision of the loxP-flanked STOP cassette. Ptgff ${ }^{C r e E R}$ mice will be characterized elsewhere. Previously published mice used in this study include: $B r n 3 a C K O A P$ (Badea et al., 2009a), R26 $6^{I A P}$ (Badea et al., 2009b), Gad2T2A-NLS-mCherry (JAX\#023140)(Peron et al., 2015), Cdx2-Cre (Coutaud and Pilon, 2013), Advillin FlpO (Choi et al., 2020), Rosa26 FSF-LSL-tdTomato (Ai65) (JAX\#021875)(Madisen et al., 2015), Rosa26 $6^{L S L-s y n a p t o p h y s i n-t d T o m a t o ~(A i 34) ~(J A X \# 012570), ~ R e t ~}{ }^{\text {CreER }}$ (Luo et al., 2009), $T_{r k B}{ }^{C r e E R}$ (Rutlin et al., 2014), TrkC $C^{C r e E R}$ (Bai et al., 2015), Pvalb ${ }^{T 2 A-F l p O}$ (JAX \# 022730) (Madisen et al., 2015), Piezo2- (Nonomura et al., 2017), and Piezo2 ${ }^{f l}$ (JAX \#027720)(Woo et al., 2014).

Mice were handled and housed in standard cages in accordance with the Harvard Medical School and IACUC guidelines. Both male and female mice were used in all experiments. For fiber fraction measurements, in vivo MEA recordings, and adult anatomical and multiphoton imaging experiments, mice were age P50-P300. Anatomical, multiphoton imaging, and in vivo MEA recordings experiments in juvenile mice were conducted between P5-P15. 


\section{METHOD DETAILS}

Acute Brainstem Slice Recordings.-Animals were transcardially perfused with icecold choline solution comprising (in $\mathrm{mM}$ ) 92 choline chloride, $2.5 \mathrm{KCl}, 1.2 \mathrm{NaH} 2 \mathrm{PO} 4,30$ NaHCO3, 20 HEPES, 25 Glucose, 5 sodium ascorbate, 2 thiourea, 3 sodium pyruvate, 10 $\mathrm{MgSO}_{4}$, and $0.5 \mathrm{CaCl}_{2}$ adjusted to $\mathrm{pH} 7.3$ with Tris Base. The brainstem and rostral spinal cord were dissected away, immersed in ice-cold choline solution and trimmed at the pons rostrally and the lower cervical spinal cord caudally. Tissue was then mounted dorsal side up and adjusted such that the surface of the dorsal column nuclei was aligned with the direction of the vibratome blade (VT1200S, Leica). An initial 25 micron slice through the remaining dura was made to permit electrode access. Subsequently, a single horizontal slice (280 $300 \mu \mathrm{m}$ thick) was cut such that it included the dorsal column nuclei and rostral spinal cord. Slices were transferred to an incubation chamber and bathed in ACSF comprising (in mM) $125 \mathrm{NaCl}, 2.5 \mathrm{KCl}, 2 \mathrm{CaCl} 2,1 \mathrm{MgCl} 2,26 \mathrm{NaHCO} 3,1.25 \mathrm{NaH} 2 \mathrm{PO} 4$, and 25 glucose, bubbled with $95 \% \mathrm{O} 2 / 5 \% \mathrm{CO} 2$ for 40-60 minutes prior to recording. Immediately prior to placing them in the recording chamber, slices were allowed to adhere to poly-L-lysine coated coverslips (15mm, Warner Instruments) and placed with their dorsal surface exposed for whole-cell recording.

Whole-cell recordings were obtained with an internal solution containing (in $\mathrm{mM}$ ): 135 Cs-methanesulfonate, 10 Hepes, 1 EGTA, 3.3 QX314 (Cl- salt), 4 Mg-ATP, 0.3 Na-GTP, 8 Na-phosphocreatine, 5 BAPTA, and 10 neurobiotin citrate, adjusted to $\mathrm{pH} 7.3$ with $\mathrm{CsOH}$. Whole-cell current-clamp recordings used an internal solution contained the following (in $\mathrm{mM}$ ): $100 \mathrm{~K}$-methanesulfonate, $11.8 \mathrm{NaCl}, 1.8 \mathrm{MgCl} 2,3.6 \mathrm{Mg}$-ATP, $0.45 \mathrm{Na}$-GTP, 12.7 phosphocreatine, 9.1 EGTA, 0.9 CaCl2, 9 HEPES, and $14 \mathrm{KOH}$. Reported voltages are corrected for a $-8 \mathrm{mV}$ liquid junction potential between the K-methanesulfonate solution and the ACSF.

Fiber fraction measurements.-Mice were anesthetized with isoflurane and placed in a small animal stereotaxic frame (David Kopf Instruments). Under aseptic conditions, the skull was exposed and two craniotomies centered at $1.5 \mathrm{~mm}$ posterior and $1.9 \mathrm{~mm}$ lateral (bilateral) from bregma were created. To retrogradely label thalamic projection neurons residing in the DCN for whole cell recordings, a borosilicate glass pipette was lowered into the thalamus to a depth of $3.1 \mathrm{~mm}$, allowed to rest for 1 minute, then retracted to $3.0 \mathrm{~mm}$. After 5 minutes, 100-200 $\mathrm{nL}$ of fluorescent retrobeads (Lumafluor) were injected at a rate of $100 \mathrm{nl} \cdot \mathrm{min}-1$ using a Microinject system (World Precision Instruments). During surgery, mice received buprenorphine and carprofen before being returned to their home cage for at least 2 days prior to slice recording. Whole-cell patch clamp recordings were obtained from fluorescently labeled thalamic projection neurons in the gracile nucleus under visual guidance. Voltage-clamped currents were recorded with an Axopatch 700A amplifier, low-pass filtered at $10 \mathrm{kHz}$, and sampled at $40 \mathrm{kHz}$ by a 16 bit A/D converter (USB-6343, National Instruments) and acquired in Wavesurfer (Janelia). Neurons were clamped at -70 $\mathrm{mV}$ and estimates of access resistance based on the height of the fast current transient during test voltage steps were 5-20 MOhm. The dorsal column was stimulated electrically with a steel bipolar electrode (30204, FHC) or optically in mice expressing ReaChR in glabrous or hairy skin innervated A $\beta$-LTMRs. To optogenetically stimulate axons, the objective was 
positioned 1-4 millimeters caudal to the gracile nucleus and the field stop adjusted to produce a $\sim 100$ micron spot of light on the ipsilateral dorsal column. Brief pulses (100-700 microsecond) of light from an LED light source (M470F3, Thor Labs) was delivered and the intensity adjusted to attain successes and failures in roughly equal proportion, thus stimulating a single fiber. Heterotypic inverse fiber fraction measurements are expressed as the ratio of the amplitude of the current evoked by saturating electrical stimulation to the optogenetic single fiber amplitude. Homotypic inverse fiber fraction measurements are expressed as the ratio of the amplitude of the current evoked by saturating optical stimulation to the optogenetic single fiber amplitude (Litvina and Chen, 2017).

Glabrous skin A $\beta$ RA-LTMRs that innervate Meissner corpuscles and hairy skin A $\delta$ LTMRs are both labeled in TrkB ${ }^{C r e E R} ; A v i F^{F l p O} ; R 26^{L s L-F s F-R e a C h R:: m C i t r i n e ~ m i c e . ~ H o w e v e r, ~}$ hairy skin A $\delta$-LTMRs do not project up the dorsal column, and thus their axons are absent in horizontal acute brainstem slices that include the GN and dorsal column. Therefore, we used this genetic labeling strategy to preferentially activate glabrous skin A $\beta$ RA-LTMR inputs to the GN using optogenetics (Supplemental Figure 6). To achieve corresponding selective genetic access to hairy skin A $\beta$ RA-LTMR inputs in brainstem slices, we noted that Pacinian corpuscle innervating A $\beta$ RA2-LTMRs, Meissner corpuscle innervating A $\beta$-LTMRs, and hairy skin A $\beta$ RA-LTMRs are all labeled by tamoxifen administration at Ell.5 in $R e t^{C r e E R}$ mice, but hairy skin A $\beta$ RA-LTMRs are the only population within this group that are not labeled by $P_{v a l b}{ }^{F l p O}$ (Supplemental Figure 6). Serendipitously, the FRT site within the neo selection cassette flanking ChR2 in Credependent ChR2 transgenic mice $R 26^{L S L-F R T-C h R 2:: Y F P-a t t B-F R T-N e o-a t t P}(\mathrm{Ai} 32 \mathrm{D})$ remains unexcised, allowing for a Cre ON, Flp OFF subtractive strategy whereby A $\beta$ RA-LTMRs

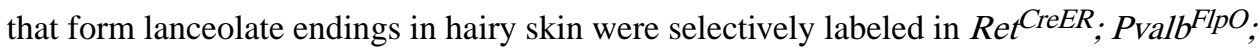
$R 26^{L S L-F-C h R 2:: Y F P-F}$ mice (Supplemental Figure 6). Therefore, we used $\operatorname{TrkB} B^{C r e E R}$;

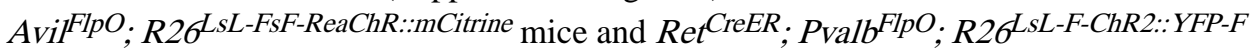
mice to selectively express an opsin in the GN terminals of either glabrous skin- or hairy skin-innervating A $\beta$ RA-LTMRs, respectively (Figure 6A; Supplemental Figure 6).

Stereotactic injections for brainstem calcium imaging.-Brainstem projection neurons were retrogradely transduced through injection of AAV2-retro viruses to their brain targets. Three to four weeks prior to imaging of the adult brainstem (or one week prior for P10-P14 imaging), mice were injected with sustained release buprenorphine (0.1 $\mathrm{mg} / \mathrm{kg}$ ), anesthetized with isoflurane and placed in a small animal stereotaxic frame (David Kopf Instruments). To target thalamic projection neurons from brainstem, the skull was exposed under aseptic conditions and two craniotomies centered at $1.5 \mathrm{~mm}$ posterior and 1.9 $\mathrm{mm}$ lateral (bilateral) from bregma were created. A borosilicate glass pipette was lowered to a depth of $3.2 \mathrm{~mm}$, allowed to rest for 1 minute, then retracted to $3.1 \mathrm{~mm}$. After 5 minutes, $100-200 \mathrm{~nL}$ of rAAV2-retro jGCaMP7s ( 1e13 gc/mL, Addgene) was injected at a rate of $70 \mathrm{~nL} \cdot \mathrm{min}-1$ using a Microinject system (World Precision Instruments). This procedure was repeated a second time to target a different location along the AP axis with a second injection in the coordinate range $1.3-1.7 \mathrm{~mm}$ posterior to bregma. To target neurons that project to the inferior colliculus, the skin was retracted under aseptic conditions and craniotomies placed bilaterally at $.5 \mathrm{~mm}$ posterior to lambda and $1.5 \mathrm{~mm}$ lateral to the 
midline. A glass pipette was lowered to a depth of $1 \mathrm{~mm}$ from the surface of the brain, then retracted to $0.7 \mathrm{~mm}$. After 2 minutes, $200 \mathrm{~nL}$ of rAAV2-retro jGCaMP7s $(\sim 1 \mathrm{e} 13 \mathrm{gc} / \mathrm{mL}$, Addgene) was injected at a rate of $70 \mathrm{~nL} \cdot \mathrm{min}-1$. This procedure was repeated at a second location along the A-P axis.

In vivo brainstem imaging surgical preparation.-Mice were anesthetized with urethane $(0.5-1.5 \mathrm{mg} / \mathrm{g})$ and chlorprothixene $(5 \mathrm{mg} / \mathrm{kg})$ and placed on a bite bar (Kopf Instruments) on a custom heated recording platform. The head was flexed downward at a 45 degree angle and an incision made in the skin and overlying muscle to expose the cervical paraspinal muscles. Next, the paraspinal muscles were retracted laterally, exposing the skull and first cervical vertebra. A hemicircular cut in the skull was made with a dental drill and a posterior section of the skull overlying the cerebellum removed. The posterior atlantooccipital membrane was carefully removed through incision directly rostral to the atlas and laterally to the occipital condyles. The dural membrane was then retracted caudally through insertion of a Bonn microprobe (Fine Science Tools) between the cerebellum and dorsal surface of the brainstem. An imaging chamber that stabilizes the brainstem and displaces the cerebellum rostrally was fabricated from a circular $3 \mathrm{~mm}$ glass coverslip (CS-3R, Warner Instruments) and flanged stainless tube (Microgroup) attached to a titanium plate and mounted to a $3 \mathrm{~d}$ micromanipulator (MMN-1, Narishige). The assembly was lowered onto the caudal brainstem just rostral to the atlas and downward pressure applied to the brainstem surface until there was no apparent motion. The assembly was then translated dorsally to displace the cerebellum, centered on the obex, and the interface between the imaging chamber and skin sealed with adhesive. Healthy long-term preparations were associated with pressures that stabilized the brainstem cardiac and respiratory motions while avoiding full exsanguination of the spinal vertebral and penetrating arteries. In a small fraction of animals, arterial elaboration over the gracile nucleus made imaging infeasible.

Multiphoton calcium imaging, mechanical stimulus, and analysis.-Two-photon calcium imaging was performed using a resonant multiphoton microscope (Scientifica) equipped with a piezoelectric objective Z-stepper (P-726, Physik Instrumente). All images were acquired using a 16X, $0.80 \mathrm{NA}, 3 \mathrm{~mm}$ WD objective (Nikon) with scan mirror excursions that produced a $510 \times 510 \mu \mathrm{m}^{2}$ field of view. Volumetric data was captured by acquiring 4-6 planes with a sawtooth scan configuration, yielding a volumetric imaging rate of $7.5-5 \mathrm{~Hz}$. Each FOV was at least $20 \mu \mathrm{m}$ below or above an adjacent FOV for cell body imaging. Excitation light was provided by a Ti:Sapphire laser ( $80 \mathrm{MHz}$, Vision S, Coherent) tuned to 910-925nm with pre-chirp dispersion compensation. Laser powers measured at the front aperture of the objective were between $15-60 \mathrm{~mW}$ depending on imaging depth. Complete coverage of the gracile nucleus typically required eight tiled multiphoton imaging volumes.

Calcium imaging time series $(512 \times 512$ pixels $)$ were initially registered to a reference image to correct for motion in the rostal-caudal and medial-lateral directions. ROIs containing putative cells were automatically detected based on their morphology and time-varying indicator fluorescence from the aligned image series and manually curated to remove extraneous cell profiles. Signals were extracted as the average value in each ROI sampled 
at the volumetric frame rate using Suite2P and corrected for signal contamination from surrounding neuropil as $F_{\text {neuropil_corrected }}(t)=F_{R O I}(t)-.7 \times F_{\text {neuropil }}(t)$. Touch stimuli were $\sim 10 \mathrm{~mm}$ long skin strokes delivered once per second from the proximal to distal direction with a soft, $1.5 \mathrm{~mm}$ wide brush (Blick Art Materials, Boston MA). Stroke was delivered for 8 seconds to a given body region with 16 second inter-trial intervals. Cells were considered responsive if their average fluorescence exceeded the baseline plus 2.5 times the standard deviation of the baseline for $30 \%$ of the stimulus period.

Dorsal Column Injections.-For dorsal column injections, mice were placed on the stereotactic frame with their neck bent at a 45 degree angle. The dorsal column was exposed between the $\mathrm{C} 1-\mathrm{C} 2$ levels through retraction of the paraspinal muscles and the overlying laminar and dural membranes removed prior to injection. For neuronal innervation density estimates, 100-200 $\mathrm{nL}$ of rAAV2/1-Cre virus (Penn Viral Core, diluted to $2 \mathrm{E} 12 \mathrm{vg} / \mathrm{mL}$ ) was injected into the dorsal column 200 microns below the spinal cord surface. For optogenetic measures of convergence, $300 \mathrm{~nL}$ of undiluted virus $(1 \mathrm{e} 13 \mathrm{gc} / \mathrm{mL})$ was injected bilaterally. Incisions were sutured and the animal was allowed to recover before being returned to its home cage for postoperative monitoring.

Skin Injections.-Mice P6 and older were anesthetized with continuous inhalation of $2 \%$ isoflurane from a precision vaporizer for the duration of the procedure (5-10 $\mathrm{min})$. The animal's breathing rate was monitored throughout the procedure and the anesthetic dose adjusted as needed. The skin region of interest was swabbed with ethanol. Injections were done with a beveled borosilicate or quartz glass pipette. Forceps were used to stabilize the skin while the needle was inserted into the dermis, injecting $0.5-2 \mu \mathrm{L}$ of virus. Mice were injected in 1-6 locations within the skin region of interest, depending on skin identity and the amount of labeling desired. Mice harboring CreER alleles along with a dual recombinase fluorescent or alkaline phosphatase reporter were injected with AAV2/1-hSynFlpO (1.37e13, Boston Children's Hospital Viral Core) to target select LTMR subtypes. Mice harboring a Cre dependent reporter allele were injected with AAV2-retro-hSyn-Cre (1.2e13, Addgene 105553), while mice harboring a Flp-dependent reporter allele were injected with AAV2/1-hSyn-FlpO (1.37e13, Boston Children's Hospital Viral Core). Wild type mice were injected with AAV2/1-hSyn-synaptophysin-TdTomato (1e14, UPenn Viral Core) or AAV2/1-synaptophysin-mScarlet (1.4e13, Boston Children's Hospital Viral Core). For all injections, a small amount of fast green (Sigma F7252-5G) in $0.9 \%$ saline was added to the virus. After injection, mice recovered from anesthesia on a warm pad while being monitored, and animals where fast green was not visible after ten minutes were not used subsequently, as this indicates subdermal spread of virus. Mice were returned to the litter once normal activity had resumed. Five weeks post-injection ( 1 week post-injection for p14 animals) animals were sacrificed by transcardial perfusion under isoflurane anesthesia. A subset of animals were screened following tissue collection to confirm that viral transduction was restricted to DRGs at somatotopically appropriate segmental levels (C7-C8 for forepaw injections, L3-L5 for hindpaw injections).

Perfusions.-Mice were anesthetized under isoflurane and transcardially perfused with $5-10 \mathrm{~mL}$ of Ames Media (Sigma) in 1xPBS with heparin $(10 \mathrm{U} / \mathrm{mL})$, followed by $5-10 \mathrm{~mL}$ 
of $4 \%$ paraformaldehyde (PFA) in 1xPBS. After perfusion, the skull, vertebral column, and central nervous system were removed and post-fixed in $4 \%$ PFA in $1 \mathrm{xPBS}$ at $4^{\circ} \mathrm{C}$ overnight. Skin samples were removed and treated with commercial depilatory cream (NAIR, Church and Dwight Co.; Princeton, NJ) for 3-5 minutes, then washed with soap and water. Skin samples were fixed overnight in Zamboni's fixation buffer with gentle agitation at $4^{\circ} \mathrm{C}$. Samples were washed $3 \times 10 \mathrm{~min}$ in $1 \times$ PBS at room temp after overnight fixation before fine dissection.

Immunohistochemistry of free-floating sections.-The brain, brainstem, and spinal cord were removed from the skull and vertebral column. The skull was cut down the midline and pulled laterally to remove. The dorsal and ventral sides of the vertebral column were removed. Then, forceps were used to remove the dorsal root ganglia (DRG) bilaterally from the vertebral column and surrounding tissue, retaining the connection to the spinal cord. This was done for all DRGs working from lumbar to cervical levels. Once all DRGs had been removed, the brain, brainstem, and spinal cord could be extracted from the vertebral column and stored in 1x PBS.

Sectioning of tissue samples was done using a vibrating blade microtome (Leica VT100S). Samples were embedded in 3\% agarose in 1x PBS. Once solidified, samples were trimmed and attached to the sectioning plate with superglue. Transverse brainstem and spinal cord sections were taken at $100 \mu \mathrm{m}$ while horizontal brain sections were taken at $200 \mu \mathrm{m}$. Tissue samples were then rinsed in 50\% ethanol/water solution for 30 minutes, followed by $3 \times$ $10 \mathrm{~min}$ in $2 x P B S$. Tissue samples were incubated in a mixture of primary antibodies in high salt Phosphate Buffer Saline (2x PBS) containing 0.1\% Triton X-1000 (2xPBSt) for 72 hours at $4^{\circ} \mathrm{C}$. Primary antibodies used in this study: Guinea pig anti-Vglut1 1:1000 (Sigma AB5905), Rabbit anti-Homer1 1:500 (CedarLane 160003SY), Goat anti-mCherry 1:500 (CedarLane AB0040-200), Chicken anti-GFP 1:500 (Aves GFP-1020), Rabbit antitRFP 1:500 (Evrogen AB233), Rabbit anti-S100 1:300 (VWR/ProteinTech 15146-1-AP), Chick anti-Neurofilament $200 \mathrm{kDa}$ 1:500 (Aves NFH). The tissue was rinsed 3×10 min with $2 x P B S$ before incubation in the secondary antibody solution for 24 hours at $4{ }^{\circ} \mathrm{C}$. The secondary antibody solution was made up in 2xPBSt and contained species-specific Alexa Fluor 488, 546, and 647 conjugated IgGs. Secondary antibodies used in this study: Donkey anti-Guinea pig Alexa Fluor 647 1:500 (Jackson Immunoresearch 706-605-148), Donkey anti-Rabbit Alexa Fluor 488 1:1000 (ThermoFisher A-21206), Donkey anti-Goat IgG Alexa Fluor 546 1:1000 (LifeTech A11056), Donkey anti-Chicken IgG Alexa Fluor 488 1:1000 (Jackson Immunoresearch 703-545-155), Donkey anti-Rabbit IgG DyLight 405 1:1000 (Jackson Immunoresearch 711-475-152). The tissue was then mounted on glass slides, coverslipped in VectaMount mounting medium (Vector labs H-5000) and stored at $4^{\circ} \mathrm{C}$ until imaging.

Immunohistochemistry of skin cryosections.-Paws were roughly dissected from perfused mice and hair was removed with a commercial depilatory cream (Nair, Church \& Dwight). Glabrous and hairy tissue was finely dissected from the paws, cryoprotected in $30 \%$ sucrose in $1 \mathrm{x}$ PBS at $4^{\circ} \mathrm{C}$ for 2 days, embedded in OCT (1437365, Fisher), frozen using dry ice, and kept at $-80^{\circ} \mathrm{C}$. The tissues were cyrosectioned $(25 \mu \mathrm{m})$ using a cryostat 
(Leica) and collected on glass slides (12-550-15, Fisher). Sections were washed 3 times for 5 minutes each with $1 x$ PBS containing $0.1 \%$ Triton X-100 (0.1\% PBST), incubated with blocking solutions (0.1\% PBST containing 5\% normal goat serum (S-1000, Vector Labs) or normal donkey serum (005-000-121, Jackson Immuno)) for 1 hour at RT, incubated with primary antibodies diluted in blocking solutions at $4^{\circ} \mathrm{C}$ overnight, washed 3 times for 10 minutes each with $0.1 \%$ PBST, incubated with secondary antibodies diluted in blocking solutions at $4^{\circ} \mathrm{C}$ overnight, washed again 4 times for 10 minutes each with $0.1 \%$ PBST (DAPI solution was included in the second wash at 1:5000 dilution), and mounted in Fluoromount-G mounting medium (Fisher 0100-01).

Multielectrode Array (MEA) Recordings.-MEAs were inserted into the L4 dorsal root ganglia (H6b-32ch or E4-64ch, Cambridge Neurotech), gracile nucleus of the brainstem (E1-16ch or H6-64ch, Cambridge Neurotech), primary somatosensory cortex (F3-32ch or E4-64 ch) or inferior colliculus (H6b-32ch, Cambridge Neurotech) with a 3-axes micromanipulator (IVM mini, Scientifica). MEA signals were high-pass filtered at $200 \mathrm{~Hz}$, amplified (RHD2132) and acquired at 20kHz (RHD2000, Intantech) for offline processing.

Spike detection and cluster assignment.-Data was converted into contiguous binary files (https://github.com/peltonen/kwik-tools) and processed using JRClust (Janelia). After pre-processing and filtering, spikes that exceeded detection thresholds defined as qq factors (RQ Quiroga, 2004) that were adjusted (4.5-6.0) based on recording site and probe. Spikes were detected and assigned to clusters corresponding to individual or multiples units with JRClust (https://github.com/JaneliaSciComp/JRCLUST). These initial clusters were manually curated to remove artifacts that did not correspond to spike waveforms, and clusters were examined and split if their PCA decompositions were not monodisperse. This collection of clusters was then examined and merge operations performed if consideration of the average spike waveform and spiking cross-correlation indicated that two clusters corresponded to the same neuron. Manual curation typically resulted in putative isolated single units with intra-cluster waveform correlations greater than 0.9 , inter-cluster waveform correlations less than 0.1 , and clusters that did not meet these criteria were not considered in the data set. Clusters computed in this manner contained units with spatially distinct receptive fields, suggesting that units were drawn from non-overlapping sources. Spikes event times for each cluster were exported and processed in Python.

Optical Skin Stimulation.-Hair was removed from the hindlimb ipsilateral to site of optical stimulation with a commercial depilatory cream (Nair, Church \& Dwight). The leg and foot were immobilized with adhesive tape and positioned underneath a custom-built optical skin stimulator. Next, an image of the animal was taken along the axis of optical stimulation through a high-pass dichroic with a monochrome camera (FLIR Systems). The stimulator mirrors were parked at their origin and the skin illuminated with low power laser light. A subsequent image of the paw and laser illuminated spot was captured. This procedure allowed for the centering and orienting of maps derived from optical stimuli onto the skin. 
The optical stimulator was constructed from a laser light source ( $445 \mathrm{~nm}, 100 \mathrm{~mW})$, a beam expander (GBE03-A, Thor Labs), galvometer scan mirrors (6210H, Cambridge Technologies), an f-theta lens (FTH-160-1064-M39, Thor Labs), and a front end dichroic (FF660-Di02-25×36 Semrock) for dividing the optical path between the stimulator and camera. The recorded latencies between optical stimulation and GN neuron spiking were consistent with direct synaptic input from A $\beta$-LTMRs after accounting for the time required to transit the dorsal column, the delay across the synapse, and the time for spike generation, but may also include indirect input from the post-synaptic dorsal column pathway.

Optical Receptive Field Determination.-A spike-triggered average based analysis was used to describe the receptive fields of mechanically sensitive neurons rendered optically responsive through expression of the channelrhodopsin variant ReaChR (Hooks et al., 2015). Optical receptive fields were estimated for each recorded unit that responded to sparse white noise optical stimulation of the skin according to the equation (cite Pillow):

$$
\operatorname{Pr}(\text { spike } \mid \text { stimulus })=\frac{\operatorname{Pr}(\text { stimulus } \mid \text { spike }) \times \operatorname{Pr}(\text { spike })}{\operatorname{Pr}(\text { stimulus })}
$$

To compute $\operatorname{Pr}($ spike $\mid$ stimulus), we first binned spikes from each unit at $1 \mathrm{msec}$ to produce a $[\mathrm{N} \times 1]$ binary vector of spike occurrences, where $\mathrm{N}$ is the number of discrete time bins encompassing the recording. Next, the stimulus was binned at each time step into a $2 \mathrm{~d}$ spatial histogram with 100 micron bin edges to produce a matrix indicating stimulation of a given location. This was subsequently flattened in column-major order to produce a $[\mathrm{M} \times \mathrm{N}]$ stimulus matrix, where $\mathrm{M}$ is the number of (binned) spatial locations and $\mathrm{N}$ is the number of discrete time bins. Next, a design matrix $[(\mathrm{M} \times \mathrm{mkt}) \times \mathrm{N}]$ containing the relevant regressors for each time bin was constructed from the stimulus matrix by collecting all space-time stimulus elements within a time window of nkt samples (Pillow). The spike triggered stimulus average with dimensions $[\mathrm{M} \times \mathrm{nkt}]$ is the product of the design matrix and the spike vector. This was used to compute the quantity $\operatorname{Pr}$ (spike $\mid$ stimulus) according to the equation above by dividing by the raw stimulus ensemble. For DRG neurons, displayed spatial receptive fields were produced from the spike-triggered average by integrating over the response window (4-6 msec) are overlayed over an image of the paw.

Craniotomies.-A circular craniotomy with a diameter of $3.1 \mathrm{~mm}$ was made over right primary somatosensory cortex (stereotaxic coordinates: $0 \mathrm{~mm}$ anterior-posterior, 1.8 $\mathrm{mm}$ lateral of bregma). A titanium headplate was cemented to the mouse's skull (C\&B Metabond) and the craniotomy covered with Kwik-sil adhesive until the day of recording. The mouse was lightly anesthetized with urethane $(.2 \mathrm{~g} / \mathrm{kg})$ and chloroprothixene $(1 \mathrm{mg} / \mathrm{kg})$, placed on a heated platform and headfixed such that the paws on the side contralateral to the craniomety were extended for mechanical stimulation.

Whole-mount alkaline phosphatase staining of the skin, spinal cord, and brainstem for sparse neuronal labeling.-We used $\operatorname{Ret}^{C r e E R}$, TrkCCreER, or $P_{t g f i} C r e E R$ to label $\mathrm{A} \beta$ rapidly adapting (RA)-LTMRs in hairy and glabrous paw skin $\left(R e t^{C r e E R}\right)$; A $\beta$ slowly adapting (SA) type I-LTMRs in hairy and glabrous paw skin $\left(T r k C^{C r e E R}\right.$ ), or A $\beta$ Field-LTMRs in hairy paw skin and a previously uncharacterized 
sensory neuron in glabrous paw skin that will be described elsewhere ( $P$ tgfr $C$ reER ) (Luo et al., 2009; Bai et al., 2015; Zheng et al., 2019)(Figure 3A-B; see Methods).To estimate relative peripheral innervation densities across different body regions, Brn $3 a^{c K O A P} /+$ mice aged 4-8 weeks (for adult time points) or 7 days (for P14 timepoint) were stereotactically injected with AAV2-retro-Cre virus (Addgene 105553) into the dorsal column at cervical levels $\mathrm{C} 1-\mathrm{C} 2$ or into the dorsal column nuclei (adult animals only) as described above. Four weeks post virus injection (1 week for P14 animals), animals were transcardially perfused using $4 \%$ paraformaldehyde. Skin and paws were cut off, hair was removed using NAIR, and skin was post-fixed in Zamboni solution at $4^{\circ} \mathrm{C}$ overnight. Spinal cords and brainstems were post-fixed in $4 \% \mathrm{PFA}, 4^{\circ} \mathrm{C}$ overnight. The next day, tissue was washed three times in $1 \times \mathrm{PBS}$ and stored in PBS at $4^{\circ} \mathrm{C}$ until further processing.

To visualize brainstem arbors by sparse labeling, three different strategies were used. To sparsely label all cutaneous neurons that send ascending projections to the dorsal column nuclei, Brn3a $a^{c K O A P} /+$ animals aged 8-15 days were injected with AAV2/retro-hSyn-Cre virus into hairy or glabrous paw skin. 4-5 weeks post virus injection, animals were transcardially perfused with $4 \%$ paraformaldehyde, and skin and CNS were collected as described above. After fine dissection, brainstems were processed for whole-mount alkaline phosphatase staining (see below). For all animals with sparse labeling of DCN arbors in the gracile or cuneate nucleus (1-4 arbors per animal), the corresponding paws were dissected and stained to identify the position and morphology of the labeled cutaneous arbor. Only neurons with somatotopically unambiguously identified peripheral arbors were used for further analysis. To label specific genetic classes of LTMRs, RetCreER or Ptgfr CreER mice were crossed to $B r n 3 a^{c K O A P /+}$ mice to generate $C r e E R /+; A P /+$ animals. Low dosage tamoxifen $(\sim 0.01 \mathrm{mg})$ was delivered by oral gavage of pregnant females at E11.5 (Ret $\left.{ }^{C r E E R}\right)$ or intraperitoneal injection of pups at P21 (Ptgfr CreER). This method yielded a low success rate of animals with unambiguous, sparse labeling in paws. Therefore, to allow for higher throughput sparse labeling of genetically identified arbors innervating specific skin regions, we generated and used TauFSFiAP, a dual Cre and FlpO-dependent AP allele. Animals containing CreER alleles ( $\operatorname{Ret}^{C r E E R}$, TrkC CreER, or Ptgfi ${ }^{C r e E R}$ ) and the Tau ${ }^{F S F i A P}$ allele were administered high doses of tamoxifen to maximize Cre-dependent recombination. Then, AAV-FlpO virus was injected to glabrous or hairy paw skin at P8-P10. 5-6 weeks post virus injection, animals were transcardially perfused with $4 \%$ paraformaldehyde and their brainstems and paws were dissected and processed for whole-mount alkaline phosphatase staining. The Tau ${ }^{F S F i A P}$ allele was also used to sparsely label neurons innervating Meissner

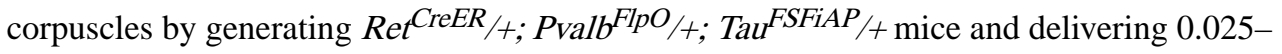
$0.05 \mathrm{mg}$ tamoxifen intraperitoneally at P5-P7.

Whole-mount alkaline phosphatase staining was performed as previously described (Liu et al., 2007). Briefly, after post-fixation, tissue was washed $3 \mathrm{x}$ in $1 \mathrm{XPBS}$, incubated at $65^{\circ} \mathrm{C}$ for 2-2.5 hours, and washed with B3 buffer (0.1 M Tris pH 9.5, 0.1 M NaCl, $50 \mathrm{mM} \mathrm{MgCl}$, $0.1 \%$ Tween-20) $3 \times 5 \mathrm{~min}$, at room temperature. AP signal was detected by incubating tissue in NBT/BCIP (Roche) diluted at $3.4 \mu \mathrm{g} / \mathrm{mL}$ in B3 buffer at room temperature, for 22-30 hrs with gentle rocking. To stop the AP reaction, tissue was incubated in 4\% PFA at room temperature for 1 hour, washed $3 \times 5 \mathrm{~min}$ in 1 XPBS, and then dehydrated ( 1 hour $50 \%$ ethanol, 1 hour $70 \%$ ethanol, 1 hour $100 \%$ ethanol followed by overnight incubation in 
100\% ethanol) before clearing with BABB (1 part benzyl benzoate:2 parts benzyl alcohol) for 4 hours at room temperature. Paws and brainstems were imaged on a Zeiss AxioZoom microscope.

Quantification of pre-synaptic boutons in the GN/CN.-To label pre-synaptic boutons of cutaneous sensory neurons, $R 26^{\text {syt-tdTomato }}$ (Ai34) animals were injected with AAV2/retro-Cre virus, or wild type animals (CD1) were injected with AAV2retro-synaptophysin-tdTomato or AAV2-retro-synaptophysin-mScarlet virus into the hairy or glabrous paw dermis at P5-P15. Animals were transcardially perfused with $4 \%$ paraformaldehyde 5-6 weeks post injection for adult time points (P50-P70), or 1 week post injection for developmental time points (P14). After overnight post-fixation in 4\% PFA at $4{ }^{\circ} \mathrm{C}$ and three washes in $1 \times \mathrm{XBS}$, dorsal column nuclei were finely dissected and sectioned transversely on the vibratome at $90-100 \mu \mathrm{m} /$ section. Antibody staining was performed as described above. Sections were mounted in VectaMount and imaged on a Zeiss LSM 700 confocal microscope using a 40x oil-immersion lens. To quantify boutons, syn-FP $\mathrm{FP}^{+}$puncta were identified semi-automatically using Imaris (Bitplane). Automatic thresholding and background subtraction were applied to all images. Total syn- $\mathrm{FP}^{+}$puncta were identified using the Spot tool with manual curation. Co-localization with vGlut1 or Homer1 puncta was assessed manually after bouton identification for a subset of images. Pre-synaptic boutons were found both at arbor end points and along the length of axonal fibers, at an equivalent density in glabrous and hairy neurons (Supplemental Figure 4).

Dual Retrograde Tracing.-AAVs encoding nuclear restricted fluorescent proteins, rAAV2-retro-hSyn-H2B-GFP-bGH (5.3E12 gc/mL) and AAV2-retro-hSyn-H2BmTagBFP2-bGH (3.17E13 gc/mL), were stereotactically injected into the thalamus and the inferior colliculus (approximately 300nl per location) of Gad2 $2^{T 2 A-N L S-m C h e r r y}$ Sections were imaged on a Zeiss LSM 700 confocal microscope. To quantify overlap of labeled nuclei, fluorescent nuclei were identified semi-automatically using Imaris. Quantification of labeled nuclei and colocalization was obtained using the Spot tool with manual curation.

Tamoxifen treatment.-Tamoxifen (Sigma T5648) was dissolved in $100 \%$ ethanol to $20 \mathrm{mg} / \mathrm{mL}$, (30 minutes vortexing at room temperature) and then mixed in $2 \mathrm{X}$ volume sunflower seed oil (Sigma S5007) and vacuum centrifuged for 30 minutes to evaporate the ethanol. $20 \mathrm{mg} / \mathrm{mL}$ tamoxifen aliquots in sunflower seed oil were stored at $-80^{\circ} \mathrm{C}$ until the day of use, when they were thawed 30 minutes at room temperature, while protected from light, and if necessary further dissolved in sunflower seed oil before administering to mice. Pregnant mice received $0.02 \mathrm{mg}-3 \mathrm{mg}$ tamoxifen by oral gavage at E11.5 (to label $\mathrm{A} \beta$ RA-LTMRs using $\operatorname{Ret}^{C r e E R}$ mice), E12.5 (to label A $\beta$ SA1-LTMRs using TrkC ${ }^{C r e E R}$ mice), or E13.5-E16.5 (to label glabrous skin innervating A $\beta$ RA-LTMRs using TrkB ${ }^{C r e E R}$ mice). To label A $\beta$ SA1-LTMRs and A $\beta$ field/free-LTMRs, $P t_{\text {gff }}$ CreER animals were administered tamoxifen postnatally by intraperitoneal injection at P21. The amount of tamoxifen delivered was titrated according to experimental needs.

In situ hybridization.-Detection of Piezo2 and Nefh transcripts was performed by fluorescent in situ hybridization, as described in (Sharma et al., 2020). Briefly, individual 
DRG ganglia were rapidly dissected from euthanized mice and frozen in dry-ice cooled 2 methylbutane and stored at $-80^{\circ} \mathrm{C}$ until further processing. DRGs were cryosectioned at a thickness of $20 \mu \mathrm{m}$ and RNA was detected using RNAscope (Advanced Cell Diagnostics) according to the manufacturer's protocol. The following probes were used: Mm Piezo2 exons 43-45 (Cat\# 439971-C3), tdTomato (Cat\# 317041), Mm-Nefh (Cat\# 443671). Sections were mounted in FluoroMount-G (Fisher 0100-01) and imaged on a Zeiss LSM 700 confocal microscope using a 40x oil-immersion lens.

\title{
Supplementary Material
}

Refer to Web version on PubMed Central for supplementary material.

\section{Acknowledgements}

\begin{abstract}
We thank Ofer Mazor and Pavel Gorelik from the HMS Research Instrumentation Core Facility, Ardem Patopoutian for Piezo2 mutant mice, Evan Feinberg and Daniel Dombeck for imaging advice, David Paul for generating viruses, Annie Chen, Sabrina Belozerova, Victoria Malarczyk, Alexandra Malarcyzk, and Connie Tsan for assistance with mouse husbandry, Rachel Essner for assistance with brainstem imaging, and Dean Eckles for statistical advice. We are grateful to Mark Andermann, Soha Ashrafi, Greg Bashaw, Chinfei Chen, Evan Feinberg, Annie Handler, Elizabeth Hong, Shan Meltzer, Aniqa Tasnim, John Tuthill, and Rachel Wilson for valuable comments. This work was supported by a William Randolph Hearst Fellowship (B.P.L), a Goldenson Fellowship (B.P.L), NIH grants F32 NS095631-01 (B.P.L.), F32-NS106807 (C.S.), K99 NS119739 (A.E.), DP1 MH125776 (C.D.H.), R01 NS089521 (C.D.H.), and R01 NS97344 (D.D.G), a HMS Dean's Innovation Grant in the Basic and Social Sciences (C.D.H. and D.D.G.), and the Edward R. and Anne G. Lefler Center for Neurodegenerative Disorders (D.D.G.). D.D.G. is an Investigator of the Howard Hughes Medical Institute.
\end{abstract}

\section{References}

Abraira VE, and Ginty DD (2013). The sensory neurons of touch. Neuron 79, 618-639. [PubMed: 23972592]

Allievi AG, Arichi T, Tusor N, Kimpton J, Arulkumaran S, Counsell SJ, Edwards AD, and Burdet E (2016). Maturation of Sensori-Motor Functional Responses in the Preterm Brain. Cereb Cortex 26, 402-413. [PubMed: 26491066]

Azzopardi P, and Cowey A (1993). Preferential representation of the fovea in the primary visual cortex. Nature 361, 719-721. [PubMed: 7680108]

Badea TC, Cahill H, Ecker J, Hattar S, and Nathans J (2009a). Distinct roles of transcription factors brn $3 \mathrm{a}$ and brn $3 \mathrm{~b}$ in controlling the development, morphology, and function of retinal ganglion cells. Neuron 61, 852-864. [PubMed: 19323995]

Badea TC, Hua ZL, Smallwood PM, Williams J, Rotolo T, Ye X, and Nathans J (2009b). New mouse lines for the analysis of neuronal morphology using CreER(T)/loxP-directed sparse labeling. PLoS One 4, e7859. [PubMed: 19924248]

Bai L, Lehnert BP, Liu J, Neubarth NL, Dickendesher TL, Nwe PH, Cassidy C, Woodbury CJ, and Ginty DD (2015). Genetic Identification of an Expansive Mechanoreceptor Sensitive to Skin Stroking. Cell 163, 1783-1795. [PubMed: 26687362]

Catani M (2017). A little man of some importance. Brain 140, 3055-3061. [PubMed: 29088352]

Catania KC (2001). Early development of a somatosensory fovea: a head start in the cortical space race? Nat Neurosci 4, 353-354. [PubMed: 11276222]

Catania KC, and Kaas JH (1997). The mole nose instructs the brain. Somatosens Mot Res 14, 56-58. [PubMed: 9241728]

Catania KC, Leitch DB, and Gauthier D (2011). A star in the brainstem reveals the first step of cortical magnification. PLoS One 6, e22406. [PubMed: 21811600]

Choi S, Hachisuka J, Brett MA, Magee AR, Omori Y, Iqbal NU, Zhang D, DeLisle MM, Wolfson RL, Bai L, et al. (2020). Parallel ascending spinal pathways for affective touch and pain. Nature 587, 258-263. [PubMed: 33116307] 
Corniani G, and Saal HP (2020). Tactile innervation densities across the whole body. J Neurophysiol 124, 1229-1240. [PubMed: 32965159]

Coutaud B, and Pilon N (2013). Characterization of a novel transgenic mouse line expressing Cre recombinase under the control of the $\mathrm{Cdx} 2$ neural specific enhancer. Genesis 51, 777-784. [PubMed: 23913642]

Cowey A, and Rolls ET (1974). Human cortical magnification factor and its relation to visual acuity. Exp Brain Res 21, 447-454. [PubMed: 4442497]

Dall'Orso S, Steinweg J, Allievi AG, Edwards AD, Burdet E, and Arichi T (2018). Somatotopic Mapping of the Developing Sensorimotor Cortex in the Preterm Human Brain. Cereb Cortex 28, 2507-2515. [PubMed: 29901788]

Drager UC, and Hubel DH (1976). Topography of visual and somatosensory projections to mouse superior colliculus. J Neurophysiol 39, 91-101. [PubMed: 1249606]

Field GD, Gauthier JL, Sher A, Greschner M, Machado TA, Jepson LH, Shlens J, Gunning DE, Mathieson K, Dabrowski W, et al. (2010). Functional connectivity in the retina at the resolution of photoreceptors. Nature 467, 673-677. [PubMed: 20930838]

Hooks BM, and Chen C (2006). Distinct roles for spontaneous and visual activity in remodeling of the retinogeniculate synapse. Neuron 52, 281-291. [PubMed: 17046691]

Hooks BM, Lin JY, Guo C, and Svoboda K (2015). Dual-channel circuit mapping reveals sensorimotor convergence in the primary motor cortex. J Neurosci 35, 4418-4426. [PubMed: 25762684]

Iwasato T, and Erzurumlu RS (2018). Development of tactile sensory circuits in the CNS. Curr Opin Neurobiol 53, 66-75. [PubMed: 29908482]

Jain N, Catania KC, and Kaas JH (1997). Deactivation and reactivation of somatosensory cortex after dorsal spinal cord injury. Nature 386, 495-498. [PubMed: 9087408]

Lee KJ, and Woolsey TA (1975). A proportional relationship between peripheral innervation density and cortical neuron number in the somatosensory system of the mouse. Brain Res 99, 349-353. [PubMed: 1182550]

Litvina EY, and Chen C (2017). Functional Convergence at the Retinogeniculate Synapse. Neuron 96, 330-338 e335. [PubMed: 29024658]

Litwin-Kumar A, Harris KD, Axel R, Sompolinsky H, and Abbott LF (2017). Optimal Degrees of Synaptic Connectivity. Neuron 93, 1153-1164 e1157. [PubMed: 28215558]

Lu CP, Polak L, Keyes BE, and Fuchs E (2016). Spatiotemporal antagonism in mesenchymal-epithelial signaling in sweat versus hair fate decision. Science 354.

Luo W, Enomoto H, Rice FL, Milbrandt J, and Ginty DD (2009). Molecular identification of rapidly adapting mechanoreceptors and their developmental dependence on ret signaling. Neuron $64,841-$ 856. [PubMed: 20064391]

Luo W, Wickramasinghe SR, Savitt JM, Griffin JW, Dawson TM, and Ginty DD (2007). A hierarchical NGF signaling cascade controls Ret-dependent and Ret-independent events during development of nonpeptidergic DRG neurons. Neuron 54, 739-754. [PubMed: 17553423]

Madisen L, Garner AR, Shimaoka D, Chuong AS, Klapoetke NC, Li L, van der Bourg A, Niino Y, Egolf L, Monetti C, et al. (2015). Transgenic mice for intersectional targeting of neural sensors and effectors with high specificity and performance. Neuron 85, 942-958. [PubMed: 25741722]

Murthy SE, Loud MC, Daou I, Marshall KL, Schwaller F, Kühnemund J, Francisco AG, Keenan WT, Dubin AE, Lewin GR, et al. (2018). The mechanosensitive ion channel Piezo2 mediates sensitivity to mechanical pain in mice. Sci Transl Med 10.

Nonomura K, Woo SH, Chang RB, Gillich A, Qiu Z, Francisco AG, Ranade SS, Liberles SD, and Patapoutian A (2017). Piezo2 senses airway stretch and mediates lung inflation-induced apnoea. Nature 541, 176-181. [PubMed: 28002412]

Olson W, Abdus-Saboor I, Cui L, Burdge J, Raabe T, Ma M, and Luo W (2017). Sparse genetic tracing reveals regionally specific functional organization of mammalian nociceptors. Elife 6 .

Packer O, Hendrickson AE, and Curcio CA (1990). Development redistribution of photoreceptors across the Macaca nemestrina (pigtail macaque) retina. J Comp Neurol 298, 472-493. [PubMed: 2229476] 
Penfield W, and Boldrey E (1937). SOMATIC MOTOR AND SENSORY REPRESENTATION IN THE CEREBRAL CORTEX OF MAN AS STUDIED BY ELECTRICAL STIMULATION1. Brain 60, 389-443.

Peron SP, Freeman J, Iyer V, Guo C, and Svoboda K (2015). A Cellular Resolution Map of Barrel Cortex Activity during Tactile Behavior. Neuron 86, 783-799. [PubMed: 25913859]

Ranade SS, Woo SH, Dubin AE, Moshourab RA, Wetzel C, Petrus M, Mathur J, Bégay V, Coste B, Mainquist J, et al. (2014). Piezo2 is the major transducer of mechanical forces for touch sensation in mice. Nature 516, 121-125. [PubMed: 25471886]

Rutlin M, Ho CY, Abraira VE, Cassidy C, Bai L, Woodbury CJ, and Ginty DD (2014). The cellular and molecular basis of direction selectivity of Adelta-LTMRs. Cell 159, 1640-1651. [PubMed: 25525881]

Schotzinger RJ, and Landis SC (1988). Cholinergic phenotype developed by noradrenergic sympathetic neurons after innervation of a novel cholinergic target in vivo. Nature 335, 637-639. [PubMed: 3262829]

Sharma N, Flaherty K, Lezgiyeva K, Wagner DE, Klein AM, and Ginty DD (2020). The emergence of transcriptional identity in somatosensory neurons. Nature 577, 392-398. [PubMed: 31915380]

Stevens CF (2002). Predicting functional properties of visual cortex from an evolutionary scaling law. Neuron 36, 139-142. [PubMed: 12367512]

Sur M, Merzenich MM, and Kaas JH (1980). Magnification, receptive-field area, and "hypercolumn" size in areas $3 b$ and 1 of somatosensory cortex in owl monkeys. J Neurophysiol 44, 295-311. [PubMed: 7411189]

Sur M, Nelson RJ, and Kaas JH (1978). The representation of the body surface in somatosensory area I of the grey squirrel. J Comp Neurol 179, 425-449. [PubMed: 417097]

Sur M, Nelson RJ, and Kaas JH (1982). Representations of the body surface in cortical areas 3b and 1 of squirrel monkeys: comparisons with other primates. J Comp Neurol 211, 177-192. [PubMed: 7174889]

Tuthill JC, and Wilson RI (2016). Parallel Transformation of Tactile Signals in Central Circuits of Drosophila. Cell 164, 1046-1059. [PubMed: 26919434]

Van Rossum G, and Drake FL (1995). Python Tutorial. Centrum voor Wiskunde en Informatica Amsterdam.

Wang L, Millecchia R, and Brown PB (1997). Correlation of peripheral innervation density and dorsal horn map scale. J Neurophysiol 78, 689-702. [PubMed: 9307105]

Wang Q, Ding SL, Li Y, Royall J, Feng D, Lesnar P, Graddis N, Naeemi M, Facer B, Ho A, et al. (2020). The Allen Mouse Brain Common Coordinate Framework: A 3D Reference Atlas. Cell 181, 936-953 e920. [PubMed: 32386544]

Woo SH, Lukacs V, de Nooij JC, Zaytseva D, Criddle CR, Francisco A, Jessell TM, Wilkinson KA, and Patapoutian A (2015). Piezo2 is the principal mechanotransduction channel for proprioception. Nat Neurosci 18, 1756-1762. [PubMed: 26551544]

Woo SH, Ranade S, Weyer AD, Dubin AE, Baba Y, Qiu Z, Petrus M, Miyamoto T, Reddy K, Lumpkin EA, et al. (2014). Piezo2 is required for Merkel-cell mechanotransduction. Nature 509, 622-626. [PubMed: 24717433]

Walcher J, Ojeda-Alonso J, Haseleu J, Oosthuizen MK, Rowe AH, Bennett NC, and Lewin GR (2018). Specialized mechanoreceptor systems in rodent glabrous skin. J Physiol 596, 4995-5016. [PubMed: 30132906]

Wu H, Williams J, and Nathans J (2012). Morphologic diversity of cutaneous sensory afferents revealed by genetically directed sparse labeling. Elife 1, e00181. [PubMed: 23256042]

Xu J, and Wall JT (1999). Functional organization of tactile inputs from the hand in the cuneate nucleus and its relationship to organization in the somatosensory cortex. J Comp Neurol 411 , 369-389. [PubMed: 10413773]

Yarbus AL (1967). Eye movements and vision (New York,: Plenum Press).

Zheng Y, Liu P, Bai L, Trimmer JS, Bean BP, and Ginty DD (2019). Deep Sequencing of Somatosensory Neurons Reveals Molecular Determinants of Intrinsic Physiological Properties. Neuron 103, 598-616 e597. [PubMed: 31248728] 
Disproportionate representation of glabrous skin emerges over postnatal development Body representation remaps over development through changes at brainstem synapses Adult mechanoreceptors in or near glabrous skin make stronger central synapses Glabrous skin mechanoreceptors still make stronger synapses absent light touch 


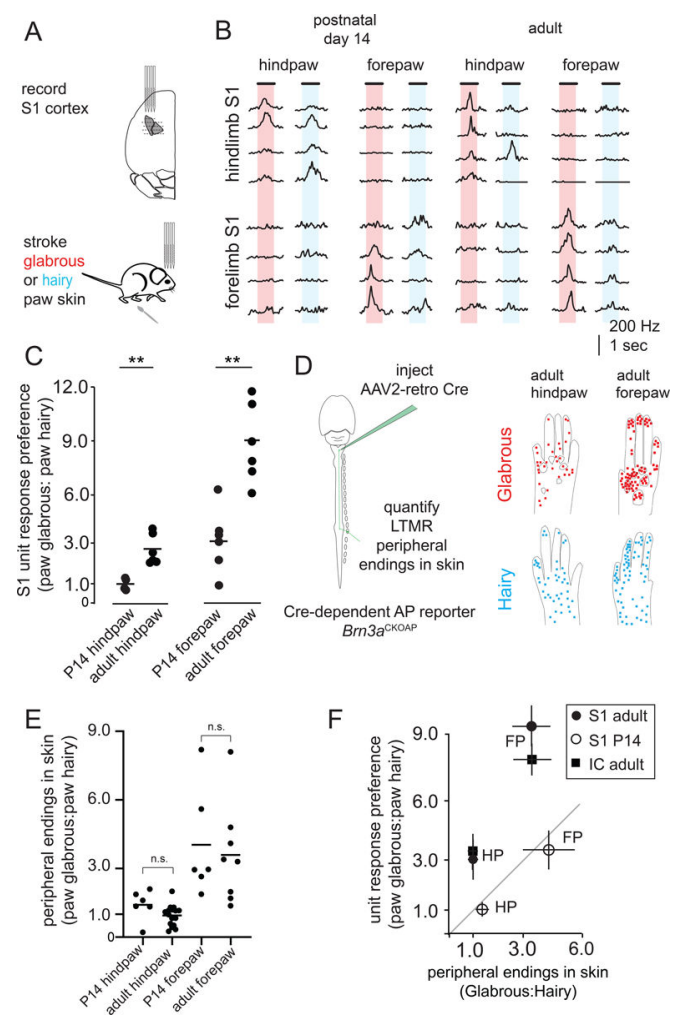

Figure 1. Central representation of glabrous skin expands over postnatal development while receptor density is stable

A. Hindlimb and forelimb S1 regions were stereotactically targeted with multiple multielectrode array (MEA) recordings in adult and postnatal day 14 (P14) mice.

B. MEA recordings from $\mathrm{S} 1$ at two developmental time points. Representative trial-averaged responses to touch of glabrous and hairy skin for hindpaw (top) and forepaw (bottom) regions of somatosensory cortex reveal a preference for glabrous skin stimulation that emerges over development.

C. The ratio of neurons preferentially responding to gentle stroke of glabrous or hairy skin for forepaw S1 and hindpaw S1. An increase in the proportion of neurons preferring glabrous skin stroke occurs over development $(n=4$ for P14 hindpaw S1, 6 for adult hindpaw S1, $\mathrm{n}=6$ for P14 forepaw S1, $\mathrm{n}=6$ for adult hindpaw S1, one-way ANOVA, $\mathrm{p}<$ 0.01 , ** Bonferroni corrected $\mathrm{p}<0.01$, Mann-Whitney U test).

D. (left) AAV2-retro-Cre virus was injected into the dorsal column nuclei to retrogradely label A $\beta$-LTMRs in Brn3APCKOAP reporter mice. A $\beta$-LTMR terminals were identified in skin to make estimates of relative innervation density. (right) Compilation of all endings identified in the paws of adult animals that received dorsal column injections, super-imposed on reference images of paws $(n=121$ neurons from 6 animals for hindpaw skin, $n=209$ neurons from 7 animals for forepaw skin). Each dot represents a single A $\beta$ LTMR terminal ending in skin.

E. The relative number of A $\beta$ LTMR endings in glabrous or hairy forepaw or hindpaw skin is stable from P14 into adulthood ( $\mathrm{n}=6$ animals for $\mathrm{P} 14$ hindpaw and forepaw, $\mathrm{n}=12$ animals for adult hindpaw, $n=7$ animals for adult forepaw, $\mathrm{p}>0.025$, Bonferroni corrected MannWhitney U test). For each paw, glabrous:hairy innervation density ratios were calculated 
by dividing the number of endings per square $\mathrm{mm}$ in paw glabrous skin by the number of endings per square $\mathrm{mm}$ in paw hairy skin.

F. (left) At P14, glabrous skin preference ratios for both forepaw (FP) S1 and hindpaw (HP) S1 are equivalent to innervation density. In adults, a greater proportion of S1 neurons respond to glabrous skin stimulation than predicted by innervation density, and a similar effect is seen in the inferior colliculus, a second major tactile area (right). Together, this demonstrates a developmental expansion of glabrous skin representation in multiple brain regions that is independent of peripheral receptor density. 


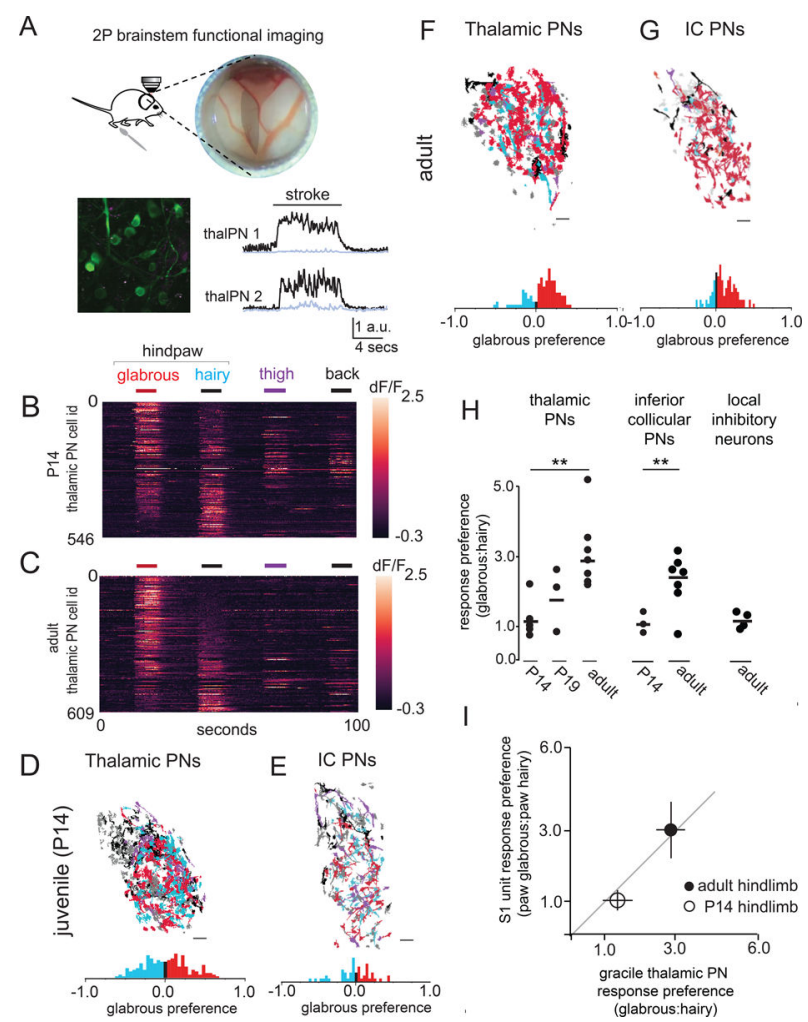

Figure 2. Disproportionate representation of glabrous skin emerges in the brainstem over postnatal development

A. (top) A preparation allowing in vivo multiphoton imaging of the gracile nucleus (GN) of the brainstem. (bottom) A representative, motion-corrected image of thalamic PNs expressing jGCaMP7, and corresponding raw fluorescence time series extracted from ROIs centered on two example neurons (black, $100 \mu \mathrm{m}$ scale bar) and surrounding neuropil fluorescence (gray) during skin stroke.

B. Touch-evoked responses of thalamic projection neurons functionally imaged in the GN. Calcium signals were evoked by stroking equivalent skin areas of the hindpaw glabrous skin (red), hindpaw hairy skin (blue), thigh (purple), and back (black) in P14 mice, data displayed is pooled across $n=3$ mice ( $100 \mu$ m scale bar).

C. Representative response to touch of the body in adult mice, similar to B.

D. Representative map of the body within thalamic projection neurons of the GN. A glabrous skin preference index, (glabrous - hairy) / (glabrous + hairy), was computed for all neurons responding to hindpaw skin stimulation in this and other experiments (bottom). At P14, there are roughly equal proportions of glabrous preferring and hairy preferring cells. E. Representative map of the body within inferior collicular projection neurons in the GN, similar to D.

F-G. Representative maps of the body in adulthood for thalamic projecting neurons (F) and inferior collicular projecting neurons $(\mathrm{G})$.

H. The proportion of thalamic or inferior collicular projecting neurons in the GN that preferentially respond to hindpaw glabrous skin touch expands over developmental time $(\mathrm{p}<$ 0.01, Mann-Whitney U test). 
I. The relationship between glabrous preference in the GN of the brainstem and glabrous preference in hindpaw S1 at two developmental timepoints. 


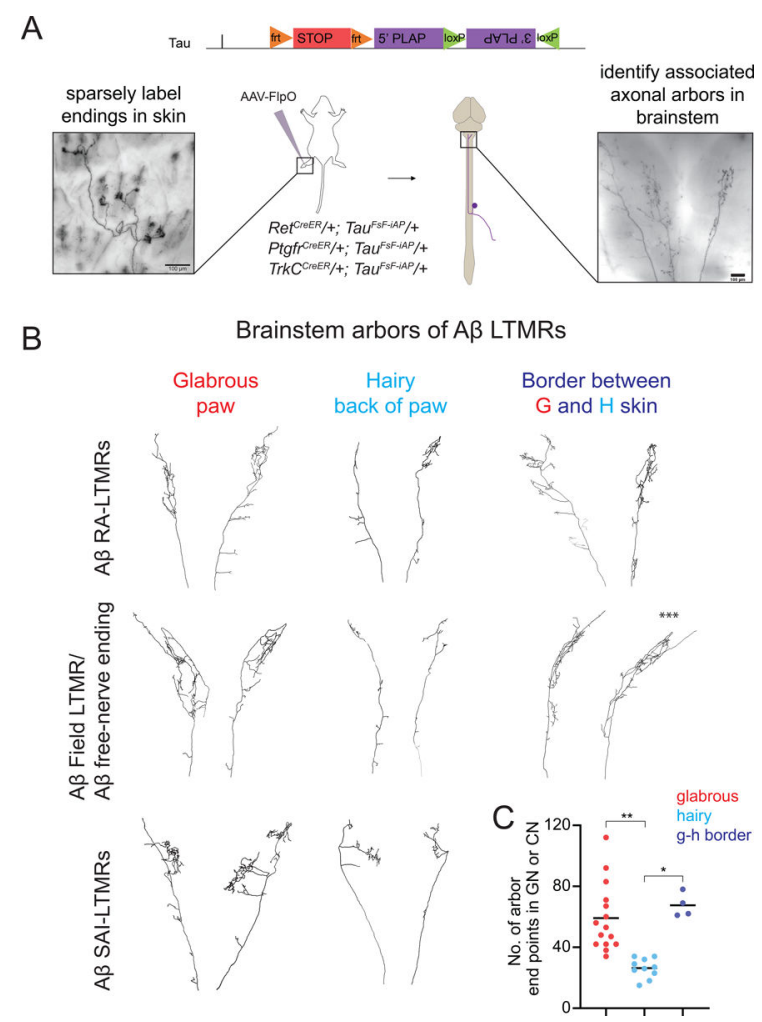

Figure 3. Target dependence of A $\beta$-LTMR central arbor morphologies.

A. Generation of a dual recombinase-dependent AP reporter mouse using the Tau (Mapt) locus ( $T a u^{F S F-i A P}$ ) for intersectional genetic labeling of A $\beta$-LTMR types with specific CreER lines and AAV2/1-hSyn-FlpO virus injection to hairy or glabrous skin. Animals with sparse labeling are analyzed to relate peripheral arbor morphologies (left) to central arbor anatomies in the $\mathrm{GN}$ or $\mathrm{CN}$ (right).

B. Representative examples of reconstructed brainstem arbors of A $\beta$ RA-LTMRs (top), A $\beta$ field/free nerve ending-LTMRs (middle), or A $\beta$ SAI-LTMRs (bottom) that innervate glabrous or hairy paw skin. Across genetic and functional classes, glabrous skin innervating neurons form more elaborate axonal arbors in the GN or $\mathrm{CN}$ than most hairy skin innervating neurons. Neurons innervating hair follicles near glabrous skin $(<0.5 \mathrm{~mm})$ form complex arbors similar to those formed by glabrous skin innervating neurons (right). A neuron that spanned both glabrous and hairy skin is indicated with asterisks (***). C. A $\beta$-LTMRs innervating paw glabrous skin or hairy follicles adjacent to glabrous skin form arbors with more end points in the GN or CN compared to A $\beta$-LTMRs innervating paw hairy skin that are more than $0.5 \mathrm{~mm}$ from glabrous skin $(\mathrm{p}<0.01$ Kruskal-Wallis one-way ANOVA, **p<0.0001 or *p<0.01, Bonferroni corrected Mann-Whitney U test), $\mathrm{n}=15$ neurons from 14 mice for paw glabrous, 10 neurons from 7 mice for paw hairy, 4 neurons from 4 mice for neurons at the edge of paw/glabrous-hairy border. 


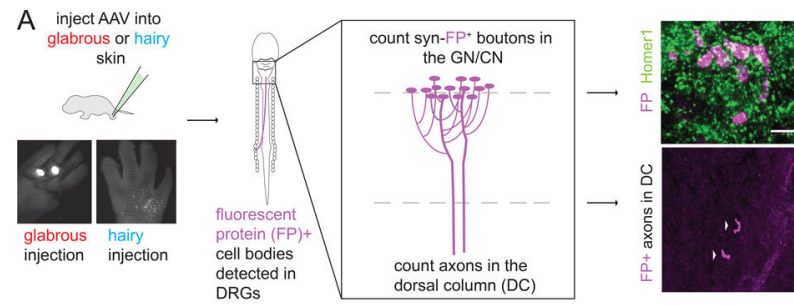

B
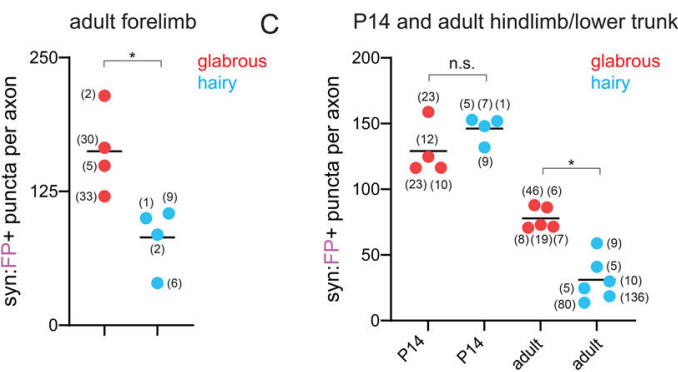

D
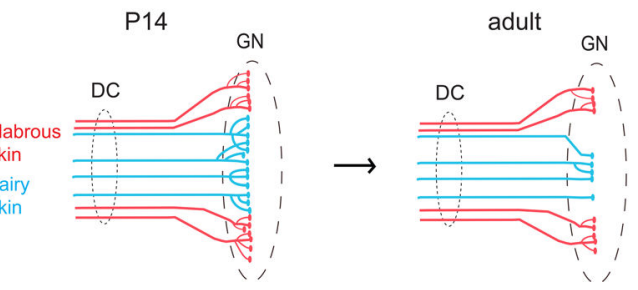

Figure 4. Relationship between skin target identity and A $\beta$-LTMR brainstem synapses over development

A. (left) AAV virus injection for skin type-specific labeling of A $\beta$-LTMR central projections with synaptophysin-tdTomato or synaptophysin-mScarlet, hereafter referred to as synaptophysin-FP (fluorescent protein). The GN and $\mathrm{CN}$ were sectioned in their entirety and stained for fluorescent proteins and synaptic markers. (right) The number of $\mathrm{FP}^{+}$presynaptic boutons and axons ascending the dorsal column were identified in each sample. Representative example of labeled boutons apposed to the excitatory postsynaptic marker Homer1 in the GN (top). Both hairy and glabrous skin-innervating A $\beta$-LTMRs form syn$\mathrm{FP}^{+}$boutons apposed to Homer $1^{+}$puncta in the GN or CN. Representative example of labeled axons (arrows) ascending the dorsal column, in a transverse section of the cervical spinal cord (bottom). Arrows point to individual axons.

B-C. Quantification of the average number of $\mathrm{FP}^{+}$pre-synaptic boutons formed by $\mathrm{A} \beta$ LTMRs innervating different skin targets. Each data point represents one animal; the number of axons identified in each animal is shown in parentheses.

B. Adult forepaw glabrous skin-innervating A $\beta$-LTMRs form more synapses than forepaw hairy skin-innervating $\mathrm{A} \beta$-LTMRs in the $\mathrm{CN}\left({ }^{*} \mathrm{p}<0.05\right.$, Mann-Whitney $U$ test).

C. At P14, hindpaw glabrous skin-innervating A $\beta$-LTMRs and hindlimb hairy skininnervating $A \beta$-LTMRs form similar numbers of pre-synaptic boutons in the GN. At adult time points, paw glabrous skin A $\beta$-LTMRs form significantly more pre-synaptic boutons in the GN than paw or trunk hairy skin A $\beta$-LTMRs ( $<<0.01$ Kruskal-Wallis one-way ANOVA, $* \mathrm{p}<0.01$, Mann-Whitney U test with Bonferroni correction for multiple comparisons).

D. Summary of skin type-dependent synaptic refinement in A $\beta$-LTMRs. 


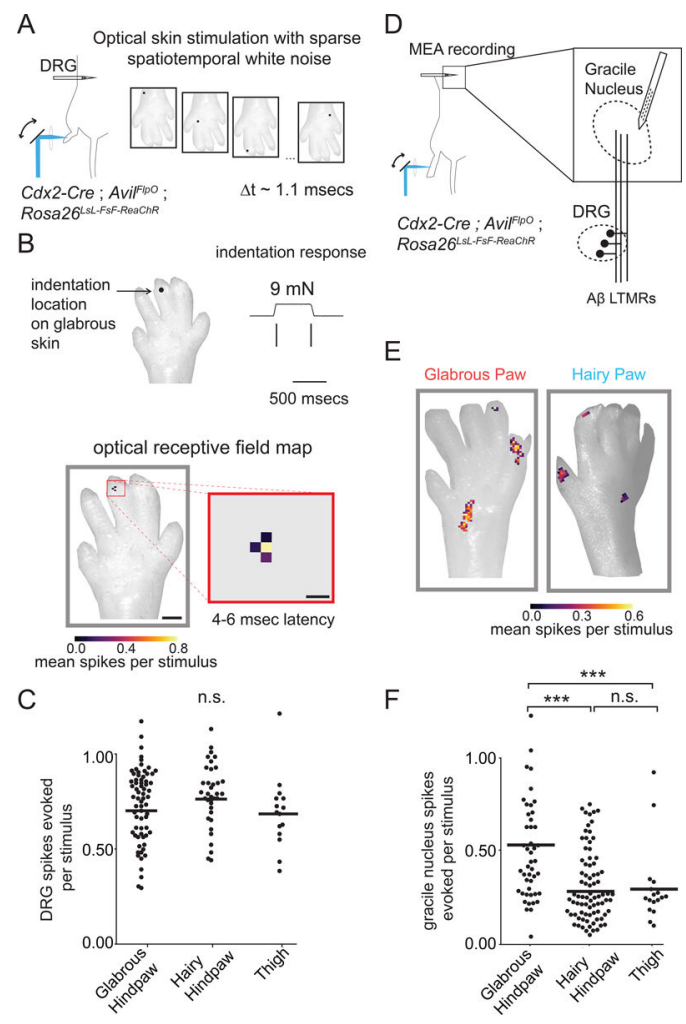

Figure 5. A $\beta$-LTMRs that innervate glabrous skin more powerfully excite central tactile neurons in the gracile nucleus than A $\beta$-LTMRs that innervate hairy skin.

A. Simultaneous in vivo recording from LTMRs with cell bodies in L4 DRG during optical stimulation of skin with sparse spatiotemporal white noise. Single pulses of focused laser light (300 microseconds, $20 \mathrm{~mW}$ ) were directed to pseudorandom skin locations every $1.1 \mathrm{msec}$ to excite ReaChR present on the terminals of cutaneous sensory neurons. Spatiotemporal optogenetic receptive fields for each unit were estimated through reverse correlation.

B. Representative single LTMR unit recorded in vivo with a MEA in the L4 DRG. (top) Spiking response to mechanical indentation for an A $\beta$ RA-LTMR. (bottom) The optogenetic spatiotemporal receptive field of the same RA-LTMR computed through reverse correlation. LTMRs have spatiotemporally simple receptive fields in skin that align with mechanical receptive fields. Scale bars are $1 \mathrm{~mm}$ and $200 \mu \mathrm{m}$.

C. The mean number of spikes evoked by a stimulus to the center of a LTMR's optogenetic receptive field, computed by integrating the conditional probability of a spike over the 3-6 msec following a light pulse. An equivalent number of LTMR spikes were evoked by stimulating optogenetic RFs in glabrous hindpaw skin, hairy hindpaw skin, and thigh skin (following hair removal), one-way ANOVA $\mathrm{p}=0.20$.

D. Strategy for exploring the functional connectivity of the GN at single LTMR resolution. Optogenetic receptive fields of touch sensitive neurons in the $\mathrm{GN}$ postsynaptic to $\mathrm{A} \beta$ LTMRs were computed similar to A-C.

E. Two representative units recorded from the GN with receptive fields on the glabrous (left) or hairy hindpaw skin (right). GN units are composed of spatially and temporally 
separable "hotspots" in their RFs, indicating convergence of multiple LTMRs onto central touch neurons in the GN.

F. The number of spikes evoked in a GN neuron by a single optical pulse to "hotspots" comprising their RFs. Optical stimulation of hindpaw glabrous skin innervating sensory neurons more effectively drives spiking in postsynaptic GN neurons than hindpaw and thigh hairy skin optical stimulation (one-way ANOVA, $\mathrm{p}<10^{-13}$, *** indicates Bonferroni corrected $\mathrm{p}<10^{-6}$ via Mann Whitney U-Test). 
A
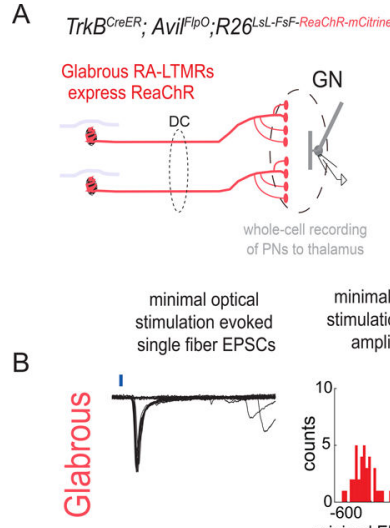

E
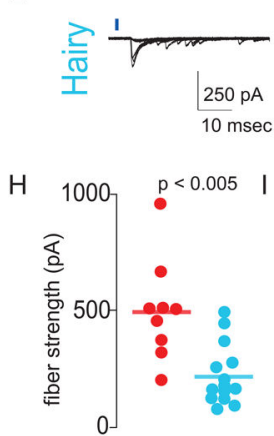

minimal optical
stimulation $E P S C$ stimulation EPSC
amplitudes
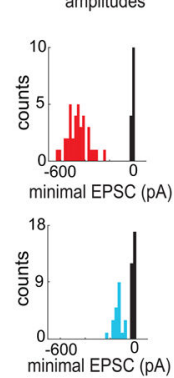

$\mathrm{F}$

C
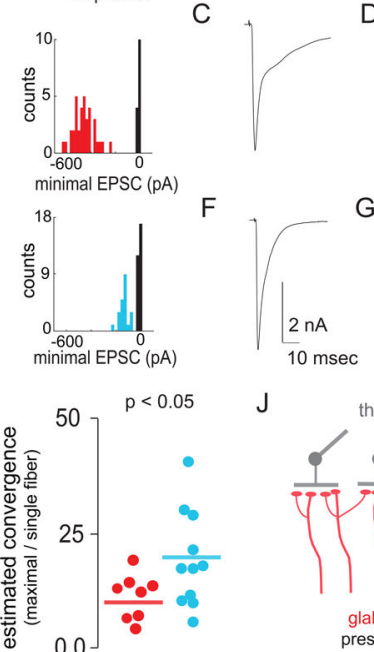

G

$\mathrm{J}$

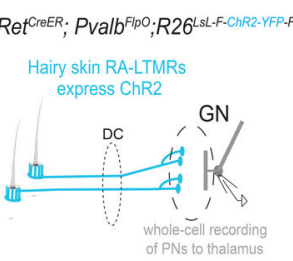

filled neurons
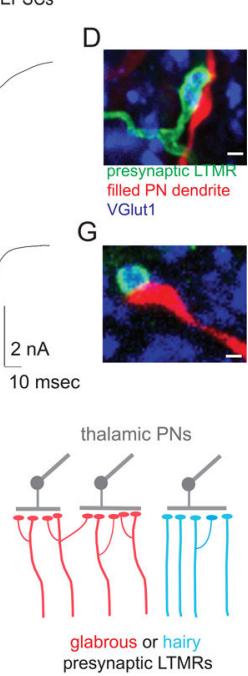

Figure 6: Synaptic strength and functional convergence of glabrous skin- and hairy skininnervating A $\beta$-LTMR synapses onto thalamic projection neurons in the brainstem.

A. Genetic strategies for assessing synaptic connections between glabrous skin- or hairy skin-innervating LTMRs and thalamic projection neurons in acute brainstem slices. (left) Glabrous skin A $\beta$ RA-LTMRs that send ascending projections through the dorsal column and synapse in the GN express ReaChR in TrkBCreER ; AviFlpO; R2 $6^{\text {LsL-FsF-ReaChR::mCitrine }}$ mice. (right) Hairy skin-innervating A $\beta$ RA-LTMRs selectively express ChR2 under a $R e t^{C r e E R}$ ON, Pvalb ${ }^{F l p O}$ OFF strategy.

B. Representative whole-cell recording from a retrogradely labeled thalamic projection neuron in the GN. (left) Minimal optical stimulation of glabrous A $\beta$ RA-LTMRs axons in the dorsal column (1-4 mm from the GN) isolated putative single fiber-evoked EPSCs as stereotyped currents accompanied by failures. (right) Histograms of EPSC amplitudes for these representative recordings are consistent with single fiber stimulation.

C. EPSC evoked by maximal stimulation of axons ascending the dorsal column, from the same recording as $\mathrm{B}$.

D. The biocytin fill of a recorded cell (red) shows apposition to VGlut ${ }^{+}$(blue), ReaChR::mCitrine+ (green) presynaptic boutons, consistent with a direct synaptic connection (scale bar: $1 \mu \mathrm{m})$.

E. Representative whole-cell recording from a retrogradely labeled thalamic projection neuron in the GN postsynaptic to hairy skin innervating A $\beta$ RA-LTMRs, similar to B.

F. EPSC evoked by maximal stimulation of all axons ascending the dorsal column in the same recording as $\mathrm{E}$.

G. Biocytin filled dendrites apposed to $\mathrm{ChR} 2: \mathrm{YFP}+$ (green) presynaptic axons, similar to D (scale bar: $1 \mu \mathrm{m}$ ). 
H. Optogenetic activation of individual glabrous skin innervating A $\beta$ RA-LTMRs neurons evokes a larger EPSC than activation of individual hairy skin-innervating A $\beta$ RA-LTMRs ( $p$ $<.005$, Mann Whitney U-test).

I. The estimated heterotypic convergence for thalamic projection neurons receiving synaptic input from glabrous skin- (red) and hairy skin-innervating (blue) A $\beta$ RA-LTMRs computed by dividing the maximal EPSC evoked by electrical stimulation of the dorsal column by the genotype-specific single fiber strength. Hairy skin A $\beta$ RA-LTMRs synaptic connections exhibit a greater estimated convergence rate than glabrous skin A $\beta$ RA-LTMRs $(p<.05$, Mann-Whitney U-test).

J. A model of differential synaptic expansion consistent with synaptic strength and convergence measurements presented in (A-I). Glabrous skin LTMR circuits thus have a lower functional convergence ratio while maintaining comparable synaptic input to thalamic PNs, resulting in synaptic expansion that enlarges the representation of glabrous skin relative to hairy skin. 


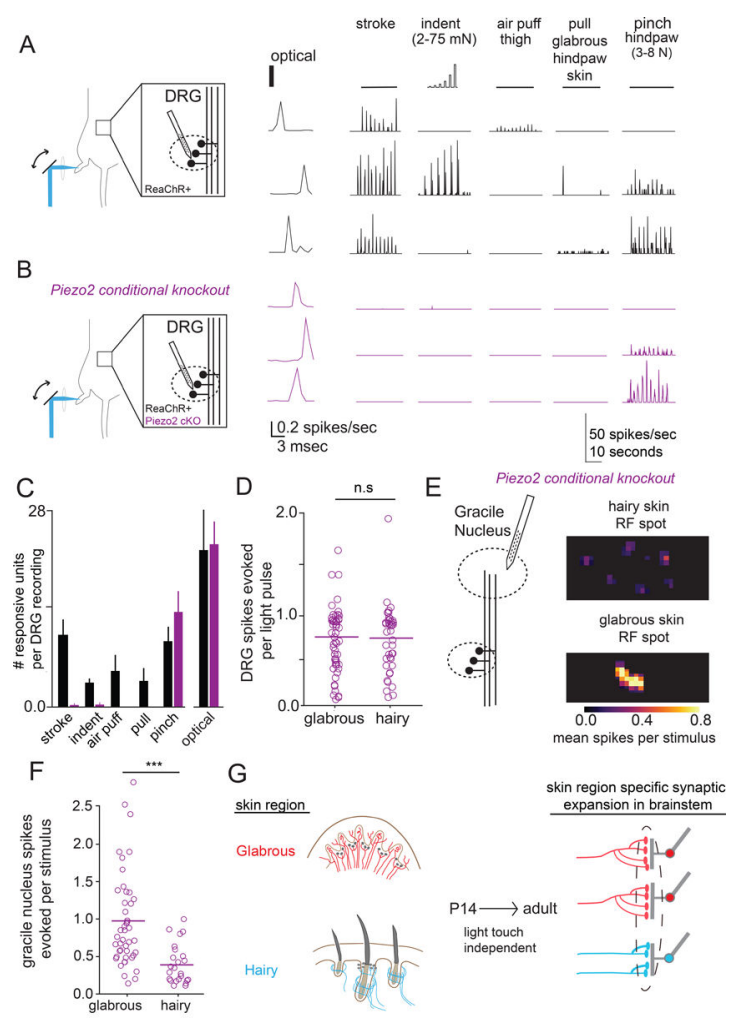

Figure 7: Disproportionate glabrous skin representation in the brainstem is maintained in mice that do not sense gentle touch

A. (left) MEA recordings from L4 DRGs in wild-type mice expressing ReaChR in all sensory neurons. (center) Representative units responded to optical stimulation of the skin with short-latency spikes. (right) Mechanical stimulation with stroke, indentation, air puff to the thigh, glabrous skin pull, and strong pinch to the hindpaw. Stroke and indentation were delivered at multiple points on the paw and thigh, and the maximal responses are displayed and quantified.

B. MEA recordings from L4 DRG in Piezo2 conditional knockout animals that express

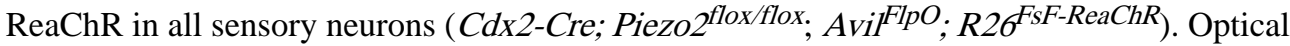
stimulation of the skin produces short-latency spikes (center), as seen in control mice (A), while responses to innocuous mechanical stimuli are largely absent (right). Responses to strong pinch to the hindpaw were preserved.

C. The number of DRG units responsive to mechanical and optical stimulation in wildtype and conditional Piezo 2 knockout mice (n=6 wt, n=5/7 Piezo $2 \mathrm{cKO}$ with mechanical/optical stimulation). Equivalent numbers of Piezo2 cKO and wildtype mouse DRG respond to noxious pinch and optical stimulation, but responses to one or more innocuous mechanical stimuli are reduced in the Piezo 2 ckO mutant (one-way ANOVA p $<.005, \mathrm{p}=0.33, \mathrm{p}=0.46$, $\mathrm{p}<.005$, respectively, Mann-Whitney U test).

D. Optical stimulation of the receptive fields of neurons innervating glabrous or hairy skin evoked equivalent numbers of spikes in Piezo2 cKO DRG neurons ( $p=0.49$, Mann-Whitney $\mathrm{U}$ test). 
E. (left) Optogenetic receptive fields were computed for neurons in the GN of Piezo2 cKO mice. (right) Representative receptive field spots of GN neurons computed after hairy skin stimulation (top) or glabrous skin stimulation (bottom).

F. The number spikes evoked in units recorded from the GN following optical stimulation of receptive field spots in hindpaw glabrous skin, hindpaw hairy skin, and thigh skin (after hair removal). Glabrous skin optical stimulation evoked more spikes in GN units than hairy skin stimulation ( $\mathrm{p}<.001$, Mann-Whitney U-test).

G. Disproportionate expansion of glabrous skin representation in the brain reflects the skin region innervated by sensory neurons independent of touch-evoked activity. 


\section{KEY RESOURCES TABLE}

\begin{tabular}{|c|c|c|}
\hline REAGENT or RESOURCE & SOURCE & IDENTIFIER \\
\hline \multicolumn{3}{|l|}{ Antibodies } \\
\hline Guinea pig anti-Vglut1 (1:1000) & Sigma & AB5905 \\
\hline Rabbit anti-Homer1 (1:500) & CedarLane & $160003 \mathrm{SY}$ \\
\hline Goat anti-mCherry (1:500) & CedarLane & AB0040-200 \\
\hline Chicken anti-GFP (1:500) & Aves & GFP-1020 \\
\hline Rabbit anti-tRFP (1:500) & Evrogen & $\mathrm{AB} 233$ \\
\hline Rabbit anti-S100 (1:300) & VWR/ProteinTech & 15146-1-AP \\
\hline Chick anti-Neurofilament 200 kDa (1:500) & Aves & NFH \\
\hline Donkey anti-Guinea pig Alexa Fluor 647 (1:500) & Jackson Immunoresearch & $706-605-148$ \\
\hline Donkey anti-Rabbit Alexa Fluor 488 (1:1000) & ThermoFisher & A-21206 \\
\hline Donkey anti-Goat IgG Alexa Fluor 546 (1:1000) & LifeTech & A11056 \\
\hline Donkey anti-Chicken IgG Alexa Fluor 488 (1:1000) & Jackson Immunoresearch & $703-545-155$ \\
\hline Donkey anti-Rabbit IgG DyLight 405 (1:1000) & Jackson Immunoresearch & $711-475-152$ \\
\hline \multicolumn{3}{|l|}{ Bacterial and Virus Strains } \\
\hline AAV2-retro-hSyn-Cre-WPRE & Addgene & 105553-AAVrg \\
\hline AAV2-retro-synaptophysin-mScarlet & Penn Viral Core & $\mathrm{n} / \mathrm{a}$ \\
\hline AAV2-retro-synaptophysin-tdTomato & Penn Viral Core & $\mathrm{n} / \mathrm{a}$ \\
\hline rAAV2-retro-hSyn-H2B-GFP-bGH & Boston Children's Hospital Viral Core & $\mathrm{n} / \mathrm{a}$ \\
\hline AAV2-retro-hSyn-H2B-mTagBFP2-bGH & Boston Children's Hospital Viral Core & $\mathrm{n} / \mathrm{a}$ \\
\hline AAV2/1-hSyn-FlpO & Boston Children's Hospital Viral Core & $\mathrm{n} / \mathrm{a}$ \\
\hline \multicolumn{3}{|l|}{ Chemicals, Peptides, and Recombinant Proteins } \\
\hline urethane & Sigma & $\mathrm{U} 2500$ \\
\hline \multicolumn{3}{|l|}{ Experimental Models: Organisms/Strains } \\
\hline TauFSFiAP & This study & $\mathrm{n} / \mathrm{a}$ \\
\hline R26-PhiC31o & JAX & $\# 007670$ \\
\hline$R 2 \sigma^{F S F-L S L-R e a C h R}$ & JAX & 024846 \\
\hline$R 26^{F S F-R e a C h R}$ & derived from JAX\#024846 & $\mathrm{n} / \mathrm{a}$ \\
\hline$B r n 3 a^{c K O A P}$ & Badea et al., 2009a & $\mathrm{n} / \mathrm{a}$ \\
\hline$R 26^{I A P}$ & Badea et al., 2009b & $\mathrm{n} / \mathrm{a}$ \\
\hline Gad2 $22 A-N L S-m C h e r r y$ & JAX & 023140 \\
\hline$C d \times 2-C r e$ & Coutaud and Pilon, 2013 & $\mathrm{n} / \mathrm{a}$ \\
\hline Advillin $^{F I p O}$ & Choi et al., 2020 & $\mathrm{n} / \mathrm{a}$ \\
\hline Rosa26 $^{\text {FSF-LSL-tdTomato }}(\mathrm{Ai} 65)$ & JAX & 021875 \\
\hline Rosa26 LSL-synaptophysin-tdTomato (Ai34) & JAX & 012570 \\
\hline$R e t^{C r e E R}$ & Luo et al., 2009 & $\mathrm{n} / \mathrm{a}$ \\
\hline $\operatorname{Trk} C^{C r e E R}$ & Bai et al., 2015 & $\mathrm{n} / \mathrm{a}$ \\
\hline $\operatorname{Trk} B^{C r e E R}$ & Rutlin et al., 2014 & $\mathrm{n} / \mathrm{a}$ \\
\hline
\end{tabular}




\begin{tabular}{|c|c|c|}
\hline REAGENT or RESOURCE & SOURCE & IDENTIFIER \\
\hline Ptgfi ${ }^{C r e E R}$ & This study; to be described elsewhere & $\mathrm{n} / \mathrm{a}$ \\
\hline$P_{\text {valb }}^{T 2 A-F l p O}$ & JAX & 022730 \\
\hline Piezo $^{-}$ & Nonomura et al., 2017 & $\mathrm{n} / \mathrm{a}$ \\
\hline $\operatorname{Piezo}^{f l}$ & JAX & 027720 \\
\hline \multicolumn{3}{|l|}{ Oligonucleotides } \\
\hline GGATGGGAAACTGAGGCTCT & Integrated DNA Technologies & $f w d-T a u^{F S F i A P}$ \\
\hline ATGGTGGCGAATTCCAAATCA & Integrated DNA Technologies & $r e V-T a u^{F S F i A P}$ \\
\hline \multicolumn{3}{|l|}{ Software and Algorithms } \\
\hline Imaris & Bitplane & RRID:SCR_007370 \\
\hline Matlab & Mathworks & $\begin{array}{l}\text { https://www.mathworks.com/products/ } \\
\text { matlab.html; RRID: SCR_001622 }\end{array}$ \\
\hline ImageJ & $\mathrm{NIH}$ & $\begin{array}{l}\text { https://imagej.nih.gov/ij/; RRID: } \\
\text { SCR_003070 }\end{array}$ \\
\hline Python & Van Rossum, G., \& Drake Jr, F. L. (1995) & https://www.python.org/ \\
\hline \multicolumn{3}{|l|}{ Other } \\
\hline NAIR hair removal cream & Church and Dwight Co.; Princeton, NJ & $\mathrm{n} / \mathrm{a}$ \\
\hline Ti:Sapphire laser & Coherent & Vision S \\
\hline CCD Camera & Rolera & Bolt \\
\hline Stereotaxic apparatus & KOPF & Model 920 \\
\hline Cannulae & Microgroup & $\mathrm{n} / \mathrm{a}$ \\
\hline UV-cured adhesive & KOA & $\mathrm{n} / \mathrm{a}$ \\
\hline Vetbond & $3 \mathrm{M}$ & $1469 \mathrm{SB}$ \\
\hline $\mathrm{C} \& \mathrm{~B}$ Metabond & Parkell & S380 \\
\hline Galvo mirrors & Cambridge Technologies & $6210 \mathrm{H}$ \\
\hline F-theta lens & Thorlabs & FTH100-1064 \\
\hline Multielectrode arrays & Cambridge Neurotech & Edge32, Edge64, and H6b \\
\hline RHD USB interface board & Intan Technologies & $\mathrm{C} 3100$ \\
\hline Multiphoton microscope & Scientifica & $\mathrm{n} / \mathrm{a}$ \\
\hline $16 \mathrm{X}$ water immersion objective & Nikon & CFI75 \\
\hline Patch-clamp amplifier & Molecular Devices & Multiclamp 700A \\
\hline
\end{tabular}

